\title{
Anomaly Detection and Classification of Physiological Signals in loT- Based Healthcare Framework
}

Menaa Nawaz ( $\square$ menaa.nawaz@students.riu.edu.pk)

Riphah International University https://orcid.org/0000-0001-6721-8010

Jameel Ahmed

Riphah International University

\section{Research Article}

Keywords: Classification, Healthcare, Internet of Things (IoT), Sensors, Filters, Signal analysis

Posted Date: June 2nd, 2021

DOl: https://doi.org/10.21203/rs.3.rs-540398/v1

License: (c) (1) This work is licensed under a Creative Commons Attribution 4.0 International License.

Read Full License 


\title{
Anomaly Detection and Classification of Physiological Signals in loT- Based Healthcare Framework
}

\author{
Menaa Nawaz, Jameel Ahmed \\ Department of Electrical Engineering Riphah International University Islamabad, Pakistan \\ menaa.nawaz@students.riu.edu.pk,jameel.ahmed@riphah.edu.pk
}

Abstract

Physiological signals retrieve the information from sensors implanted or attached to the human body. These signals are vital data sources that can assist in predicting the disease well before time and thus proper treatment can be made possible. With the addition of Internet of Things in healthcare, real-time data collection and pre-processing for signal analysis has reduced burden of inperson appointments and decision making on healthcare. Recently, Deep learning-based algorithms have been implemented by researchers for recognition, realization and prediction of diseases by extracting and analyzing the important features. In this research real-time 1-D timeseries data of onbody non-invasive bio-medical sensors have been acquired and pre-processed and analyzed for anomaly detection. Feature engineered parameters of large and diverse dataset have been used to train the data to make the anomaly detection system more reliable. For comprehensive real-time monitoring the implemented system uses wa velet time scattering features for cla ssification and deep learning based autoencoder for anomaly detection of time series signals for assisting the clinical diagnosis of cardiovascular and muscular activity. In this research, an implementation of IoT based healthcare system using bio-medical sensors has been presented. This paperalso aims to provide the analysis of cloud data acquired through bio-medical sensors using signal analysis techniques for anomaly detection and timeseries classification has been done for the disea se prognosis in real-time. Wavelet time scattering based signals classification accuracy of $99.88 \%$ is achieved. In real time signals anomaly detection, $98 \%$ accuracy is achieved. The average Mean Absolute Error loss of 0.0072 for normal signals and 0.078 is achieved for a nomaly signals.

Keywords-Classification; Healthcare; Internet of Things (IoT); Sensors; Filters; Signal analysis.

Abbreviation: Internet of Things (IoT); ConvolutionalNeural Network (CNN); Electrocardiogra m (ECG); Heart rate variation (HRV); Electromyogram (EMG); Mean Absolute Error (MAE)

\section{Introduction}

Physiological signals carries the information of the electrical activity taking place in parts of a human body [1]. Traditionally, this information is analysed by healthcare workers to realize the physiological condition of the patients and the analysis helps them in decision-making [2]. These decisions have far reaching consequences on diagnosis, treatment monitoring, drug efficacy tests, and quality of life. Physiological signals such as Electrocardiogram (ECG) carries information of human heart's electrical activity and its analysis helps in understanding the cardiova scular health of the patient [3]. The recording has been done by conventionally attaching the leads to the surface of human body over specific areas. The graph is then examined by expert healthcare professionals in order to identify the abnormalities or a nomalies. The inclusion of compute vision technology and deep learning algorithm specifically, has made the process of ECG anomaly detection, a matter of few minutes. The significance of automated detection has been validated in this Covid-19 pandemic time when personalvisits to a hospital is riskier than the problem itself. This research is an extension of our already published work where we have implemented and analyzed an IoT based healthcare system and important features from the biomedical sensor's data have been extracted by applying the signal processing techniques for anomaly detection [4]. 
In the recent years, Internet of Things (IoT) has emerged as one of the most essential communication paradigm [5]. IoT can connect number of sensors, people, vehicles, gadgets, and appliances together on internet and make the exchange of information easier and quicker. This exchange results in a large a mount of usefuldata that is accessible and can be analysed for a pplication purpose and making lives easier. IoT can assist healthcare industry due to its access to the information because of its ability to ensure connectivity. IoT applications in healthcare have helped people keep track of their medical requirements such as reminding them of appointments, keeping a check on calorie count, variations in blood pressure, a check on exercises, and many more [6]. IoT-based healthcare system field comprises of sensors, microcontrollers and other electronic devices. These devices a re capable of communicating with each other. The data received from these sensors (which are mostly bio-medical sensors) is stored in cloud for analysis and monitoring done by medical professionals or attendants. Integrating the IoT structures into medical devices advances the quality and service of care for aged patients and also for children [7]. The on-body non-invasive sensors monitors the physiological activities of human body and the information is stored and analysed for the realization, detection and potential treatment of diseases. This technology can be the breakthrough in the healthcare industry in terms of quick and safe responses, less physical contact, multiple treatment options, cost reduction and most importantly a vailability of better healthcare to the masses.

Over the past few years, there has been a lot of advancement in the design of IoT, which is spurring the use and development of smart systems in various bio-medical related processes as well. Ultimately, it has supported the improvement of healthcare system by making it intelligent and manageable. There are a lot of examples describing the effectiveness of smart healthcare including tracking of patients/biomedical equipment, a utomatic identification, correct prescription of drugs for patients and monitoring of physiological parameters of patients in real-time etc. For implementation, three of the most common smart technologies a vailable are Radio Frequency Identification (RFID), Ultra-High-Frequency (UHF) and Wireless Sensor Network (WSN). [8]

In today's digital world, Artificial Intelligence especially Deep Learning algorithms have influenced every data driven field including healthca re. Deep learning algorithms have great potential to a nalyse the data which would a ssist the medical staff in perception, recognition and prediction of disea ses and would also help in decision-making. According to a survey (Journal of Patient Safety, 2014), about 400,000 people have died in US due to wrong diagnosis and decision-making errors of practitioners. Deep learning algorithms can reduce the diagnosis time and cost and would also help in providing accurate treatment. Convolution NeuralNetwork [9] has emerged as the most frequently used algorithm for extracting important features from the data mainly due to its ability to generate good quality featured trained da ta automatically, and requires the least data pre-processing. Recently, $1 \mathrm{D}$ CNNs have not only managed to achieve high-quality results over 2D CNNs while training temporal data [10], but have also shown better performance with less complexity in both the computations and the training methods. Time-series classification of patient's physiological data helps to recognise and realize the anomaly in the physiological features by learning and identifying the temporal patterns of the acquired real-time data. Temporal data has temporal abstraction and patterns and learning the important features for the classification of time series data is a problem that has been addressed by researchers in the past few years. TSC utilizes the temporalorder of the data to analyze, each time the task involves human cognition [11]. All these applications make TSC a desirable algorithm for dealing with real-time data analysis. The process of classification of a time series involves labelling or a ssigning a cla ss to the data/time series.

In this research, design and implementation of an IoT-based health monitoring framework is presented, where real-time data from on-body sensors have been acquired and stored on cloud for the purpose of real-time monitoring as well as analyzing for the anomaly detection. The featured engineered data is then trained for timeseries classification using 1D CNN. In a case study where we have acquired some signals of bio-medical sen sors for monitoring, the vital such a selectrocardiogram (ECG), heart-beat and electromyogram (EMG) have been analysed as they are important indicators of physiological health. Also, the system is connected to cloud for transferring, recording and classification of healthcare data, which can assist other healthcare professionals for study purpose as well as patients to self-monitor their vitals. The implemented system is quick in generating the warnings if any anomaly is detected and cla ssifying the type of anomaly which would make decisionmaking easier for the medical professional in treating the patient immediately.

In the remaining part of this article, we present the related study in section II, design and implementation in section III; Signal Processing of acquired data and time series classification is given in section IV, based on analysis of signal data, results a re discussed in the section IV. We then conclude the article in section $\mathrm{V}$. 


\section{Related Work}

Temporal data has temporal abstraction and patterns and learning the important features for the classification of time series data is a problem that has been addressed by researchers in the past few years. Time series classification groups the temporal data on the basis of extracted features into classes for identification and recognition. In this research an IoT based healthcare framework is given with real-time feature extraction and $1 \mathrm{D} \mathrm{CNN}$ has been applied to learn and trained the extracted data for timeseries classification. Preprocessing and feature extraction through applying signal processing techniques would make the feature extraction process much reliable. Researchers have invested a good a mount of time in a nalyzing signal processing techniques for the construction of a Structural Health Monitoring (SHM) for damage detection [12]. A simple Butterworth filter has been used to de-noise the signals Cross-correlation to detect the extent of damage. Detection and classification of physiological signals like ECG for heartbeat diagnosis that has utilized Discrete Wavelet Transform (DWT), and Support Vector Machine (SVM), has resulted in 98.8\% classification accuracy [13]. The limitation of such work is usually the vector size. Some other aspects including security of Industrial IoT based healthcare has been explored with the inclusion of encrypted techniques like water-marking for the prevention of theft. Large amount of data collected by body sensors in IoT based healthcare should be managed properly. For this reason, Big Data analytic techniques have been introduced in healthcare organizations [14]. Deep learning algorithms have the capability for analysing big and varied datasets, as compared to machine analysis and classification methods. This is because Deep learning algorithms have the ability to avail all the a vailable input during development process [1]. Biomedical signals including EEG, ECG, EMG etc. have been analyzed for this purpose. In another research [15][16] a healthcare system for critical patients and this system is to be operated by medical attendant/nurse anywhere. System has a smart phone application developed to operate with bio-medical sensors for acquiring the required data, and dedicated health server. Authors have collected different types of bio-signals from patients using the developed system in order to monitor and analyse the data received from bio-sensors. The designed system has its own limitation as the biometric information is being transmitted to the smartphone using Bluetooth, making it low range, and then the acquired data is sent to the dedicated health server for analysis, also an encrypted form of this analysed data is stored in the database.

The process of classification of a time series involves labelling or assigning a class to the data/time series. As the accessibility of temporaldata has been increased [17], a large number of algorithms have been proposed to deal with the classification problem. Generally, TSC can be categorized into feature based, model based and distance-based methods [11]. Most commonly feature based classification methods include discrete wavelet transform (DWT) and principal component analysis (PCA) [18]. While Markov method and hidden markov method (HMM) lies under the category of model-based classifica tion and distance of time series has been mea sured by dynamic time wrapping (DTW) in some cases. Traditionalmethods covers assigning the labels to nearest neighbors (NN) or k-Nearest Neighbor for multivariate time series[19], in some cases combination of NN and DTW have produced good results [20]. Over the past few years with the advancement in deep Neural networks, deep learning algorithms have been introduced to solve the time series classifica tion problems. Algorithms such as recurrent neural networks (RNN) and 1-D convolutional neural networks (CNNs), have been shown to provide state-of-the-art results on challenging activity recognition tasks with little or no data feature engineering, instead using feature learning on raw data [21]. In deep learning algorithms the Auto Encoder (AE) is an unsupervised way of learning the features of the training data [22]. It is a type of a neural network whose prime function is to reconstruct the input data as an output. By unsupervised it is meant that $\mathrm{AE}$ has the ability to learn the important input features by itself, by utilizing the non-linear mapping without even labelling the data, and then reconstruct the input data. Convolutional Auto Encoder is modified type of AE where 1D-CNN layer is integrated to extract the important features from raw input data while 2D-CNN layer is added forimage processing. As we have mentioned above that this study is specifically for 1D raw ECG data, we will focus on 1-D CNN integration with AE (CAE) in biomedical signal processing applications. AE comprises of an encoder part and a decoder part in its algorithm. The $\mathrm{CNN}$ layers help in compressing the input data following the convolutional layers, pooling layers and by using different kernel sizes [23]. Unlike conventional CNNs, CAE are trained to minimize the loss ratio for decreasing reconstruction error during training stages on encoder/decoder. 


\section{SYSTEM DESIGN \& IMPLEMENTATION}

This aim of this work is to design and implement an IoT- based healthcare system having, uniqueness as its capability to combine and enable different, yet complementary, technologies. Basically, the system we have pictured should have the ability to collect and analyze real- time signals/data about the physiological parameters of a patient and deliver them to a signal processing unit. It then on finding anomaly in patient's data sends alerts to a medical professional, helping patients taking active role in managing their health. Figure 1 shows the design of IoT-Based healthcare system and signal analysis in the same framework. In this system, signals from sensors connected to a patient are transferred to Arduino (NodeMCU ESP8266). NodeMCU ESP8266 is connected to PC via mini USB port. NodeMCU ESP8266 is equipped with Atmega 328 microcontroller and compatible with the Arduino IDE with wifi SoC. It belongs to the Boards Manager selection of ESP-12 family, has six extra GPIOs as compared to ESP-12E module [24].

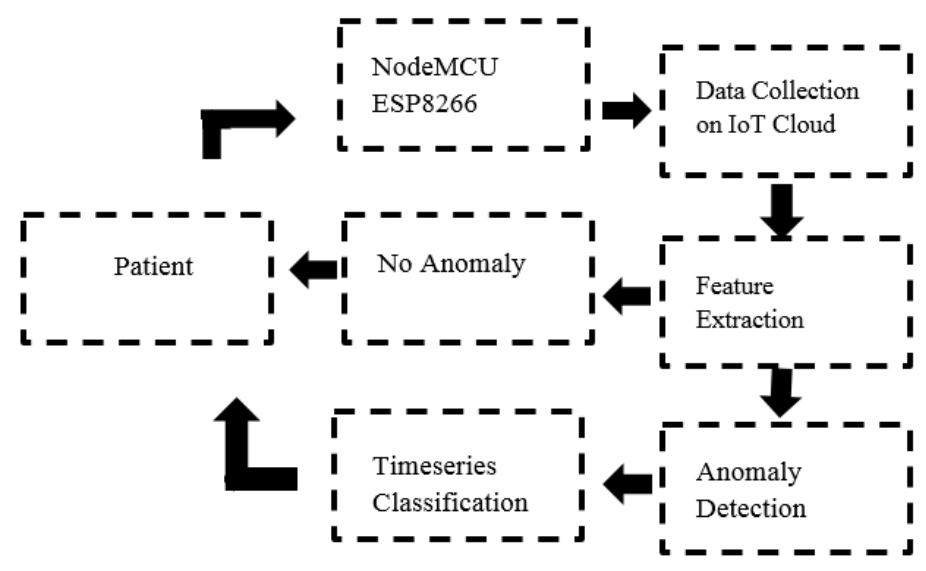

Figure 1:Figure 1 Block Diagram of IoT-Based Healthcare

System with Signal Processing and anomaly detection

Adafruit IO is an open source platform a vailable for data logging with MQTT library. Sensors data is displayed and logged on to the relevant feeds which can be private and public. In public mode data can be viewed by anyone in the world connected to internet while in private mode data is viewed by the owner of the feed only, giving him required privacy.

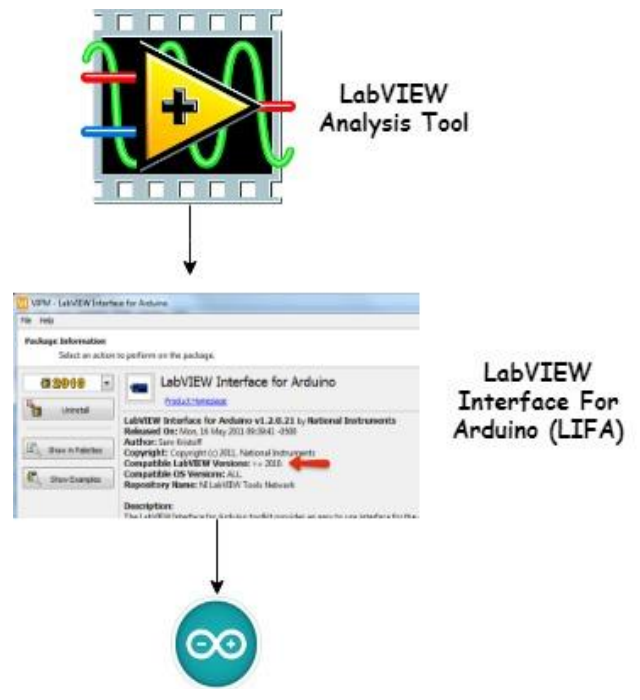

Figure 2: System Integration for Signal Analysis 
Online data is retrieved and processed in LabVIEW for anomaly detection. The Arduino is acquired in LabVIEW through NI NISA (Virtual Instrument Software Architecture VISA) and LabVIEW Interfacing For Arduino (LIFA) which is a standard for configuring, programming, and troubleshooting instrumentation system s comprising GPIB, VXI, PXI, Serial, Ethernet, and/or USB interfaces. Data is fed through serial port interface to LabVIEW for online signal processing.

\section{Signal processing}

\section{A. ECG Signals:}

Electrocardiogram (ECG) is the measurement of body's electrical potentials. For the mea surement, contact electrodes are placed on the surface of body. There are different factors causing distortions in the measured signals including patient's movement, contact a rea between skin and electrodes and breathing, generally known as baseline wanderings. Baseline wanderings is a noise signals which can mask important features of ECG signal. Certain methods can be used to remove the artifacts in ECG signals, generally categorized into major two types of filtering, non-adaptive and adaptive filtering. In this paper, wavelet adaptive filtering is used for the elimination of ECG's baseline wanderings to minimize the distortion. Coefficients of the transform and filtered output have been used in order to reconstruct the signal, and then ba seline-removed signal has been produced using inverse wavelet transform.

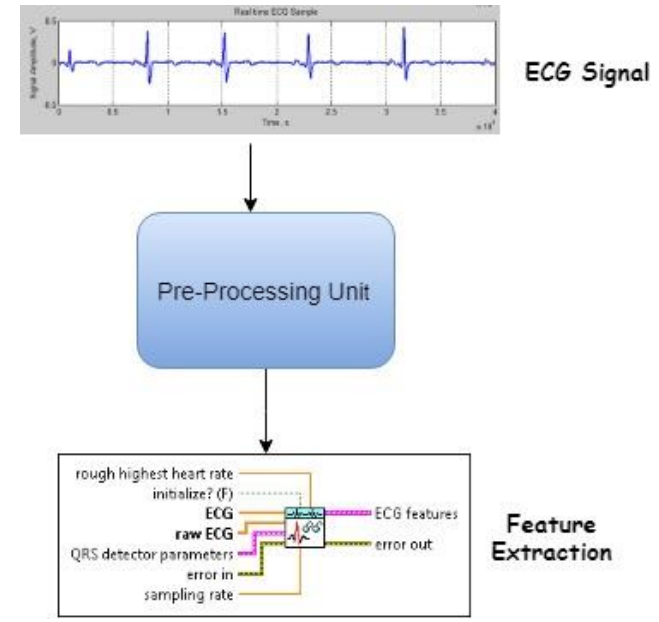

Figure 3:Signal processing steps in ECG analysis

\section{B. Heart Rate Variability (HRV):}

Heart rate variability (HRV) is the measure of the variation between successive heartbeats over period of time. It relies on heart rate regulation. HRV is an important feature reflecting physiological factors and the normal heart rhythm. It also provides an understanding of the interaction between the sympathetic and parasympathetic nervous systems. Therefore, Heart Rate Variation analysis has become a popular noninvasive tool for evaluating the nervous system and its a utomatic activities. [25].

\section{Electromyogram (EMG) Signals:}

Electromyography (EMG) is the study of neurophysiologic mechanisms of fatigue in human body muscles. It's a non-invasive tool that's helps in the mea surement of the muscle's activation level in myofibrils membrane [26]. Fatigue can be seen as reduction in muscle force. Signal processing of EMG signal using frequency domain, helps in extracting important features such as its dispersion values (standard deviation (SD) and variance measures), the median power frequency (MPF), and 
the slope, or the rate of decrease, of the MPF. In this research all of these variables have been extracted and analysed to measure the state of human muscle fibers.

\section{Wavelet Scattering Transform}

The wavelet transform computes the inner products of a time series signal [27]. Transient in electrocardiogram (ECG) signal is present due to presence of Heart Beat. These transients generally are not smooth and are of short duration. Transients are precisely captured by wavelets due to its flexibility in shape and short duration. ECG time-series signals usually require higher frequency resolution as compared to time resolution because they have low-frequency component. Whereas high time resolution is required for High-frequency components in ECG because they vary quickly with time. Therefore, a multiresolution analysis method would analyze an ECG signal precisely if the ECG signal comprises of both High and Low-frequency components. In wa velet scattering, the propagation of data is done by a series of wavelet transforms, nonlinearities, and averaging. Therefore, it produces a low-variance representations of time series. Wavelet time scattering yields signal representations insensitive to shifts in the input signal without sacrificing class discriminability.
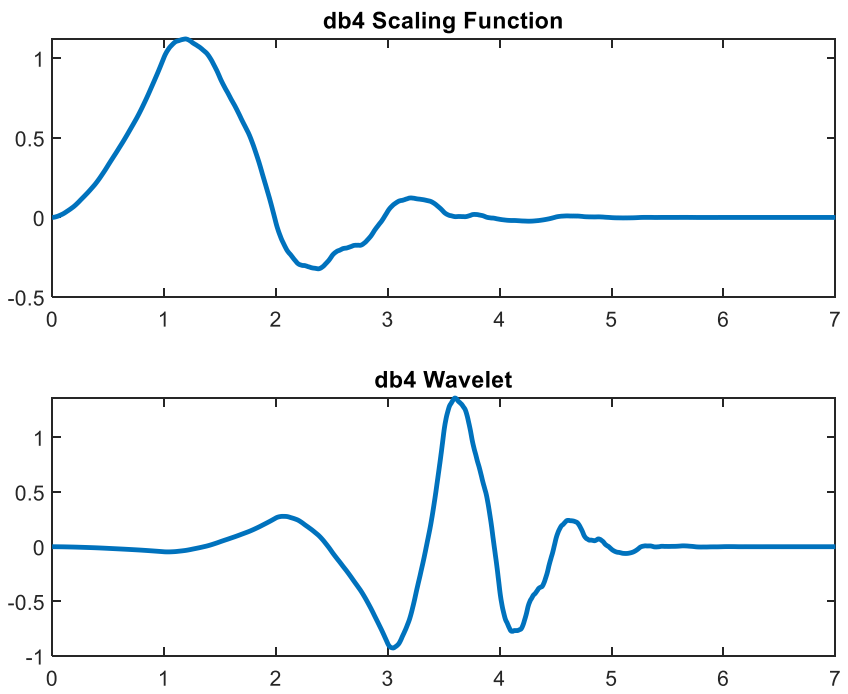

Figure 4: Wavelet Transform and Scaling Function
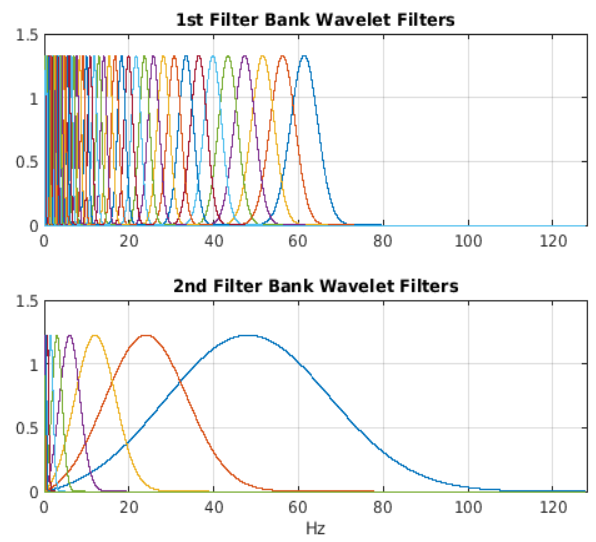

Figure 5: 1st Filter and 2nd Filter Bank Wavelet Filters [28]

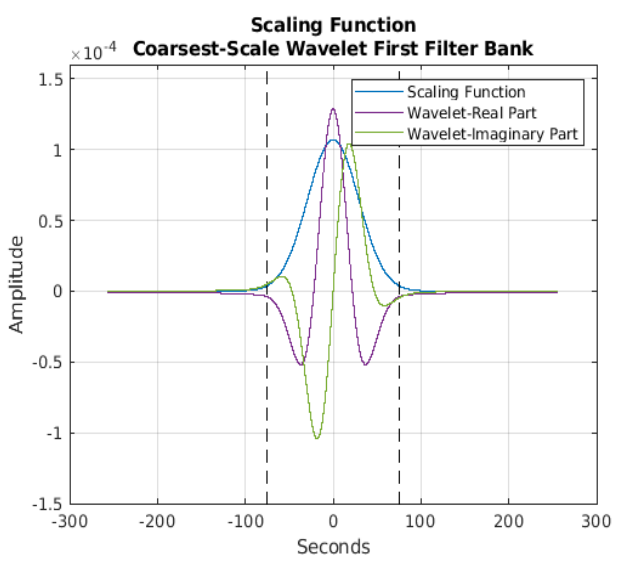

Figure 6: Coarsest-scale Wavelet First Filter Bank[28] 


\section{Simulation Results \& Discussion}

In the designed IoT based hea lthcare framework discussed in previous section, data acquisition has been done. The acquired data is filtered in the first step of analysis, which includes de-trending and de-noising of the acquired bio-signals to accurately compare the minimum threshold values already computed into the designed framework. Figure 7 shows the unfiltered ECG signal, to make it free from baseline wanderings and other motion artifacts, Wavelet transform has been used. Wavelet transform has its own significance due to its better localization properties in both time and frequency domain. Decomposition of a normalECG signal wa veform comprises of $\mathrm{P}$ wave, QRS complex and $\mathrm{T}$ wave. These are the important parameters we have located during the analysis of detected ECG signal. In order to understand the cycle for better analysis, $\mathrm{P}$ wave relates to the heart's atrial depolarization and the $\mathrm{T}$ wave corresponds to heart's ventricular repolarization.

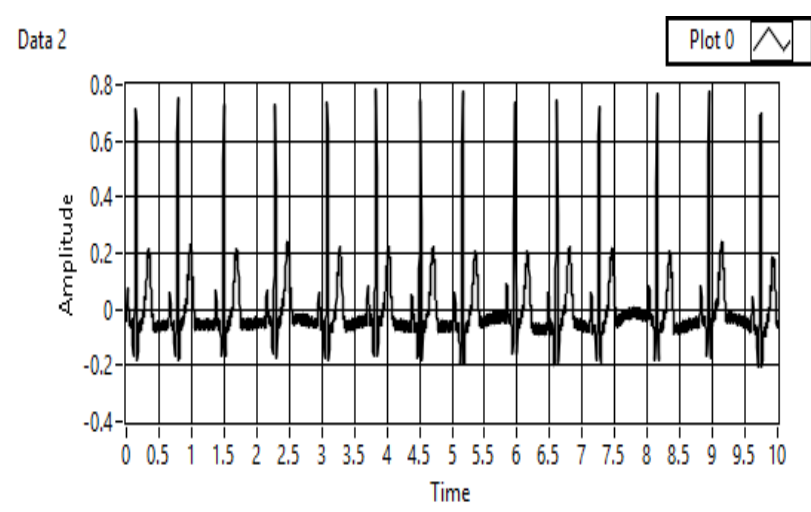

Figure 7: Unfiltered ECG Signal

Wavelet transform is applied to remove noise or motion artifacts in ECG signal shown in figure 8. Data 3 $\operatorname{Plot} 0 \backslash$

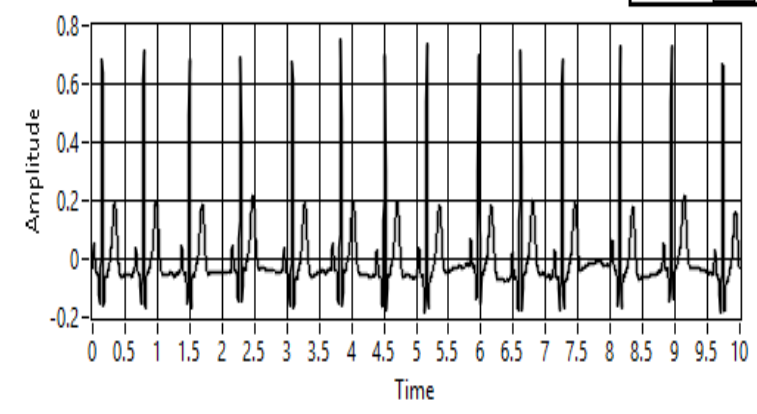

Figure 8: De-trend \& De-noised ECG signal

Table 1 shows the information of located intervals and peak values which are compared to threshold normal values (stored manually) for generating medical alerts. The ECG data is received via biosignal acquisition device and stored on the cloud in 512 values per second. If the variation of LabVIEW detected particular interval is large, irregular ECG is detected. The R-R intervaland QRS interval serve as important indicators of various heart diseases, such as arrhythmia. 


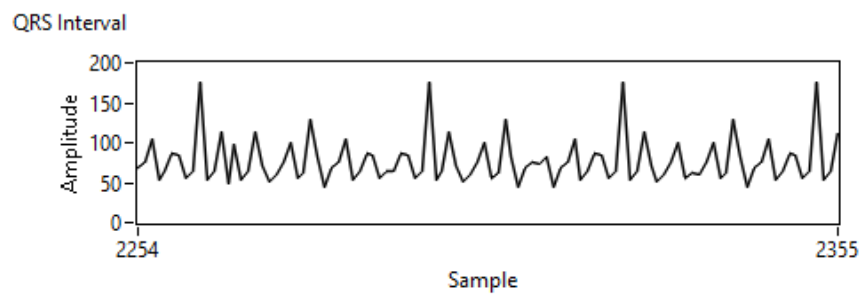

Figure 9: Peak and Intervaldetection in ECG Signal

Next variable that is part of our analysis is HRV. This is variation in time between succesive heart beats and is in control of a basic part of our nervous system called the autonomic nervous system (ANS). An abnormalHRV pattern leads life-threatening cardiac diseases like arrhythmias.

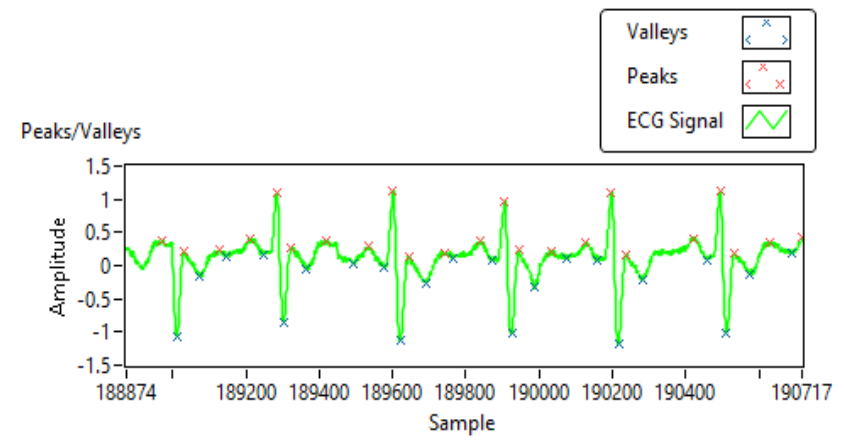

Figure 10: Detection of QRS interval

Table 1: Normal values and their Standard Deviation

\begin{tabular}{|c|c|c|c|c|c|}
\hline $\begin{array}{l}\text { Heart Rate } \\
\text { Mean (bpm) }\end{array}$ & $\begin{array}{l}\text { QRS Amplitude } \\
\text { Mean (mv) }\end{array}$ & $\begin{array}{l}\text { QRS Time } \\
\text { Mean (s) }\end{array}$ & $\begin{array}{l}\text { PR Interval } \\
\text { Mean }\end{array}$ & $\begin{array}{l}\text { QT Interval } \\
\text { Mean (s) }\end{array}$ & $\begin{array}{c}\text { ST level } \\
\text { Mean }(\mathrm{mV})\end{array}$ \\
\hline
\end{tabular}

\begin{tabular}{|c|c|c|c|c|c|c|}
\hline 78.06 & 0.86 & 0.062 & 0.13 & 0.338 & -0.035 & -0.294 \\
\cline { 2 - 7 } $\begin{array}{c}\text { Heart Rate } \\
\text { Std }(\text { bpm })\end{array}$ & $\begin{array}{c}\text { QRS Amplitude } \\
\text { Std (mv) }\end{array}$ & $\begin{array}{c}\text { QRS Time } \\
\text { Std (s) }\end{array}$ & $\begin{array}{c}\text { PR Interval Std } \\
(\mathrm{s})\end{array}$ & $\begin{array}{c}\text { QT Interval } \\
\text { Std(s) }\end{array}$ & $\begin{array}{c}\text { ST level Std } \\
(\mathrm{mV})\end{array}$ & $\begin{array}{c}\text { Iso Level } \\
\text { Std(mV) }\end{array}$ \\
\cline { 2 - 7 } & 0.84 & 0.002 & 0.009 & 0.009 & 0.027 & 0.079 \\
\hline
\end{tabular}

$\begin{array}{ccccccc}\text { Heart Rate } & \text { QRS Amplitude } & \text { QRS Time } & \text { PR Interval } & \text { QT Interval } & \text { ST level } & \text { Iso Level } \\ \text { Mean }(\text { bpm }) & \text { Mean }(\mathrm{mv}) & \text { Mean }(\mathrm{s}) & \text { Mean } & \text { Mean }(\mathrm{s}) & \text { Mean }(\mathrm{mV}) & \text { Mean }(\mathrm{mV})\end{array}$

Table 2: Tachycardia Detection Mean (s) Mean

(s)

\begin{tabular}{|c|c|c|c|c|c|c|}
\hline 119.89 & 1.182 & 0.145 & 0.13 & 0.34 & -0.213 & 0.21 \\
\cline { 2 - 7 } $\begin{array}{c}\text { Heart Rate } \\
\text { Std }(\text { bpm })\end{array}$ & $\begin{array}{c}\text { QRS Amplitude } \\
\text { Std (mv) }\end{array}$ & $\begin{array}{c}\text { QRS Time } \\
\text { Stdan(s) }\end{array}$ & $\begin{array}{c}\text { PR Interval } \\
\text { Std(s) }\end{array}$ & $\begin{array}{c}\text { QT Interval } \\
\text { Std(s) }\end{array}$ & $\begin{array}{c}\text { ST level Std } \\
(\mathbf{m V})\end{array}$ & $\begin{array}{c}\text { Iso Level } \\
\text { Std(mV) }\end{array}$ \\
\cline { 2 - 7 } 0.98 & $\mathbf{0 . 0 1 1}$ & $\mathbf{0 . 0 0 7 8}$ & $\mathbf{0 . 0 2 3}$ & $\mathbf{0 . 0 5 9}$ & $\mathbf{0 . 0 3 8}$ & $\mathbf{0 . 0 2 5}$ \\
\hline
\end{tabular}




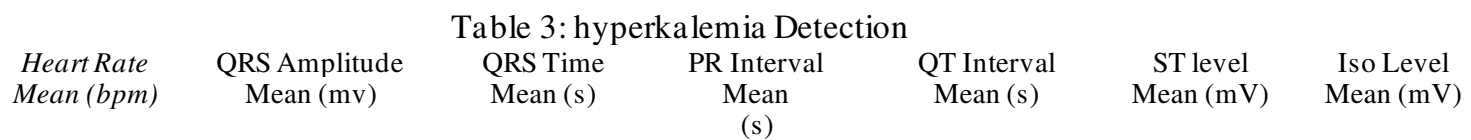

\begin{tabular}{|c|c|c|c|c|c|c|}
\hline 84.07 & 0.635 & 0.056 & 0.141 & 0.339 & 0.11 & -0.326 \\
\hline $\begin{array}{l}\text { Heart Rate } \\
\text { Std(bpm) }\end{array}$ & $\begin{array}{l}\text { QRS Amplitude } \\
\text { Std (mv) }\end{array}$ & $\begin{array}{c}\text { QRS Time } \\
\text { Stdan(s) }\end{array}$ & $\begin{array}{l}\text { PR Interval } \\
\text { Std(s) }\end{array}$ & $\begin{array}{l}\text { QT Interval } \\
\text { Std(s) }\end{array}$ & $\begin{array}{c}\text { ST level Std } \\
(\mathrm{mV})\end{array}$ & $\begin{array}{l}\text { Iso Level } \\
\text { Std(mV) }\end{array}$ \\
\hline 0.85 & 0.018 & 0.002 & 0.012 & 0.008 & 0.021 & 0.062 \\
\hline
\end{tabular}

HRV can be effected by other factors as well including aging and gender. Figure 12 reflects heart rate number under normalconditions.

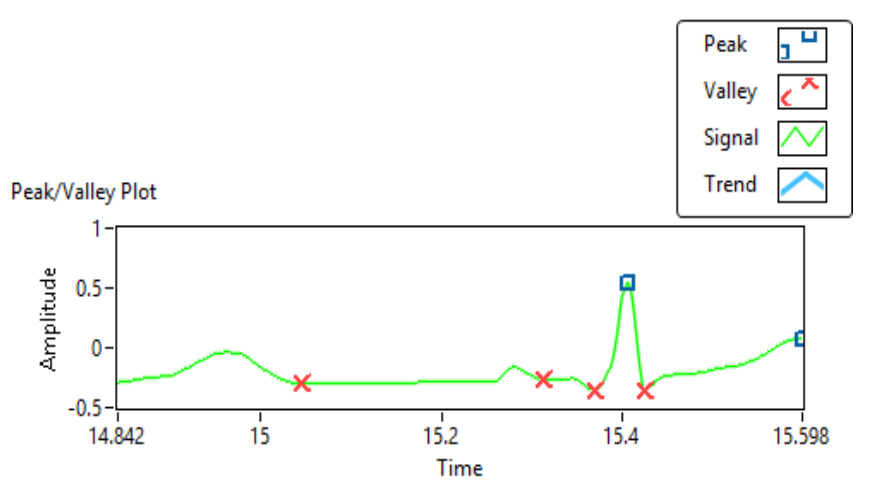

Figure 11: Peak/Intervaldetection in ECG Signal

HRV

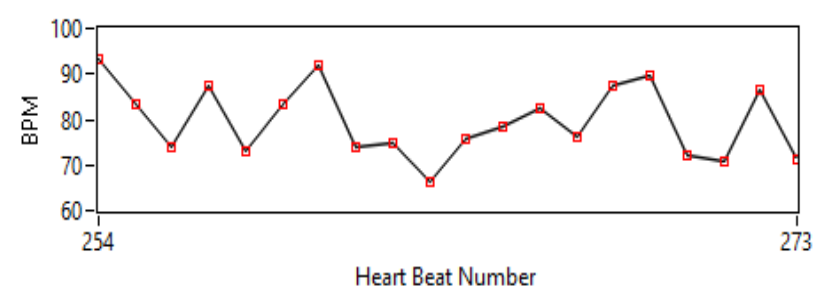

Figure 12: Mea surement of HR

Another variable which is part of our analysis is ploting of EMG signal for detection of abnormalities. A $0.5 \mathrm{~Hz}$ EMG signal shown in figure 13 is acquired from bio-signal sensor and is stored on cloud forfurther analysis and anomaly detection. 


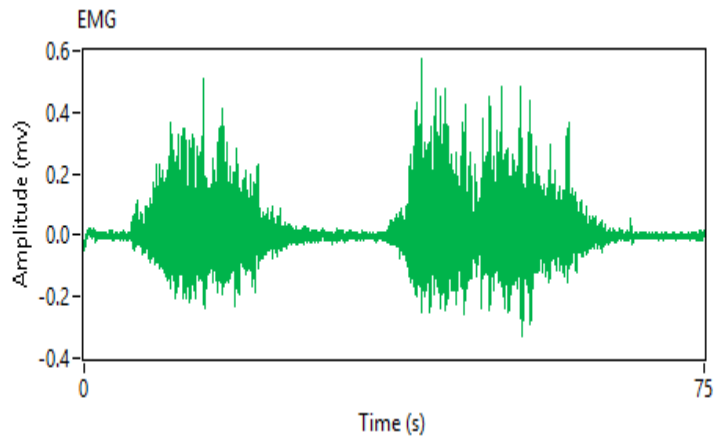

Figure 13: EMG Signal

Studies have shown the significance of EMG signals analysis in the frequency domain in order to get useful intuition about muscle fiber. We have used the frequency spectrum to generate other mea sures a ssociated with EMG frequency analysis. The aim is to identify and validate the frequency domain beha vior of EMG signal and so to determine median, a vera ge and mod frequency para meters from the power density spectrum to get useful information about the muscle state.

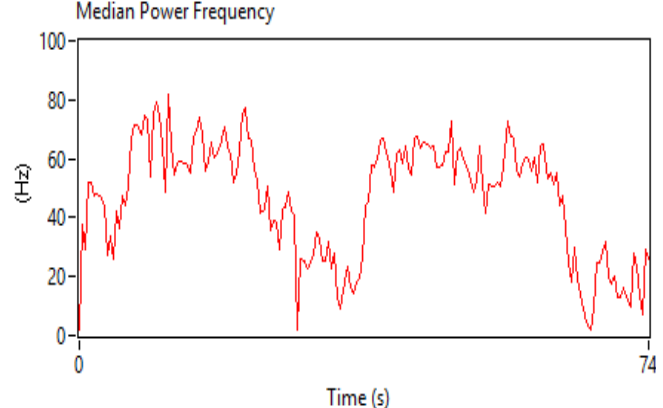

Figure 14: Median Power Frequency of EMG Signal

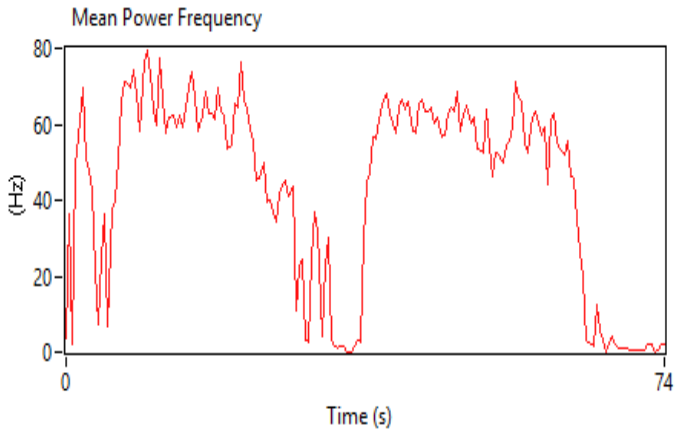

Figure 15: Mean Power Frequency of EMG Signal

\section{Wavelet Time Scattering}

Wavelet time scattering network required key parameters are the time invariant scale, the number of wavelet transforms, and the number of wa velets per octa ve in wa velet filter banks. In this research, the two cascaded filter banks are used. The wavelet time scattering network consists of default filter banks. The invariance scale is set to $150 \mathrm{sec}$ and sampling frequency is set to $128 \mathrm{sec}$.

\section{A. Input Data}

Physiological signals used in this research are collected from Physionet[29]. Three datasets are used in this research including MIT-BIH Arrhythmia Database, MIT-BIH Normal Sinus Rhythm Database and The BIDMC Congestive Heart Failure Database. Data has three classes arrhythmia (ARR), congestive heart failure (CHF), and normal sinus rhythm (NSR) with total 162 records. Training data is structured into array of two filed with data and label. The data field is $162 \times 65536$ matrix where each row is a training recording sampled at $128 \mathrm{~Hz}$. Sample Plot of each class is shown in fig 16 to 18 . 


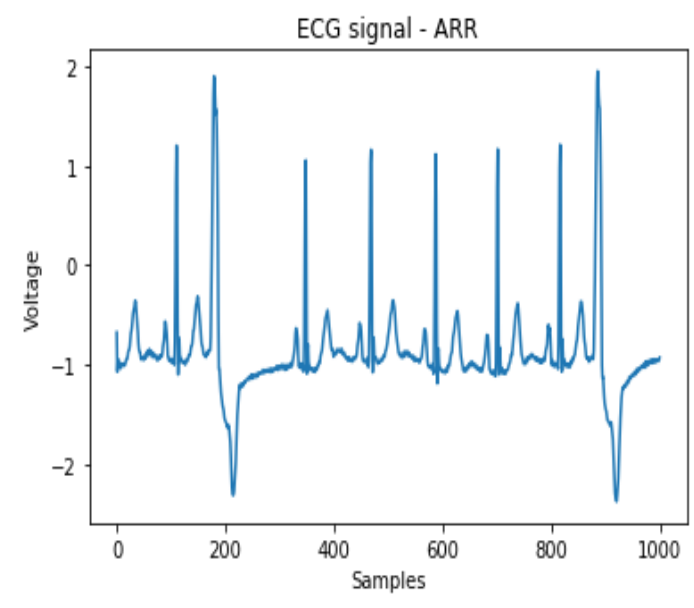

Figure 17: ECG Signal for Arrythmia

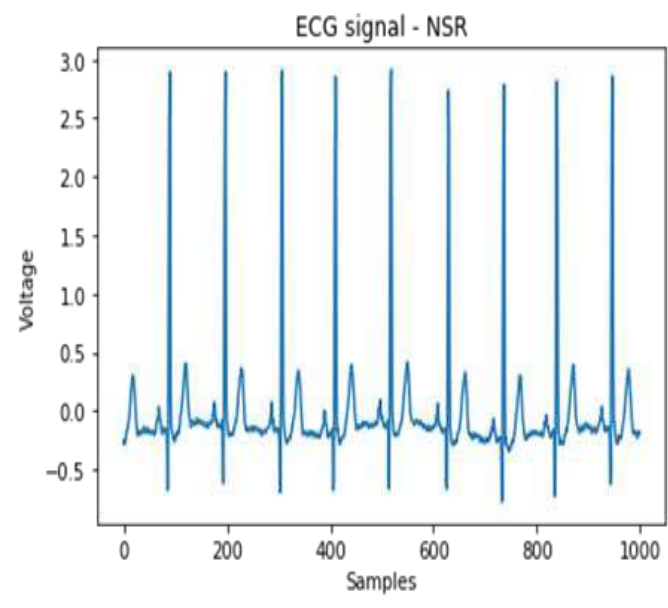

Figure 16: ECG Signal for Normal Sinus rhythm

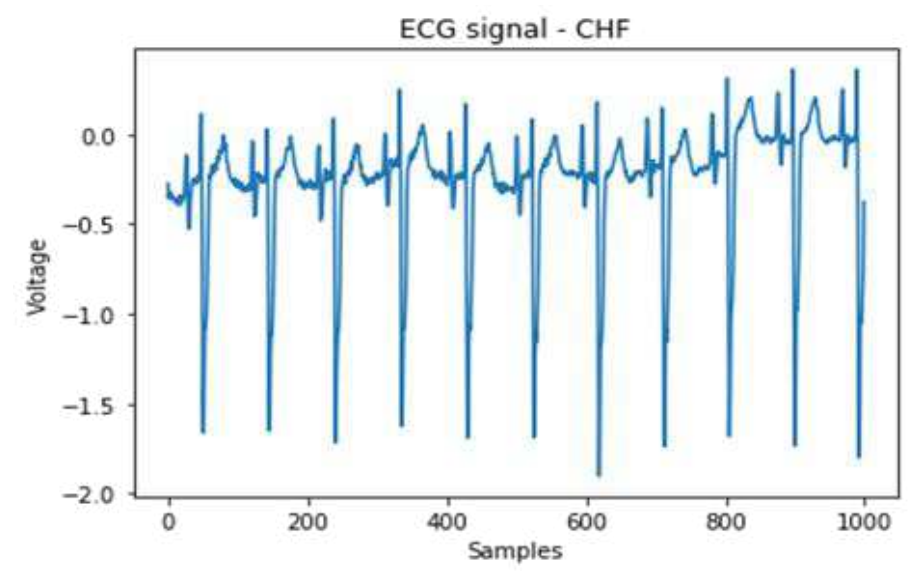

Figure 18: ECG Signal for Congestive Heart Failure

The zeroth and first order coefficients are plotted in figure 19,20. The all-scattering sequence data is passed into multiclass SVM to classify the signals. The overall $99.88 \%$ accuracy is achieved on scattering sequences over complete da ta set. For second a nalysis on train and test data, we fit a multiclass SVM to the $80 \%$ training data and tested the model for predictions on the $20 \%$ of the test data. There are 32 data records in the test set. The a chieved accuracy is $98 \%$. The corresponding confusion matrix is shown in figure 21 . 


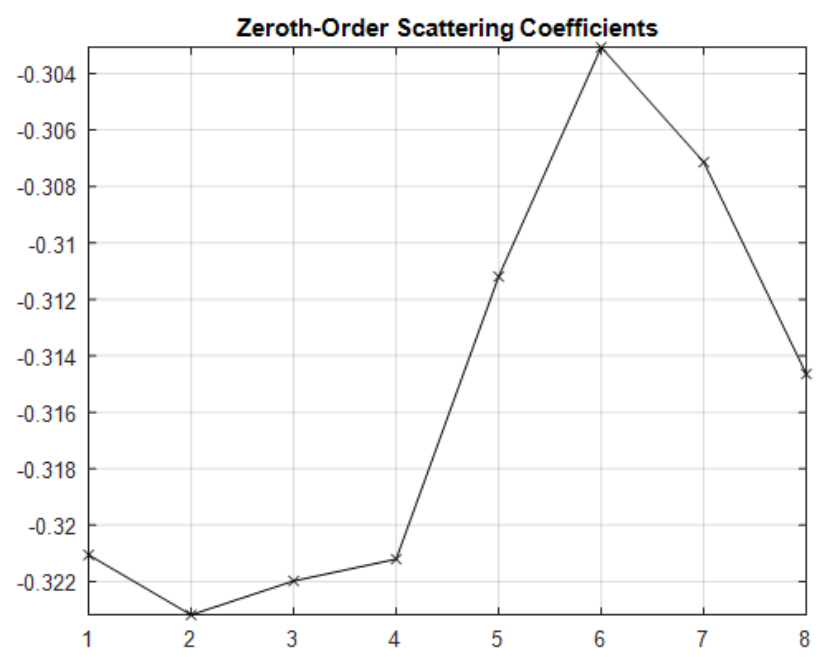

Figure 19: Zeroth-order Scattering Coefficients

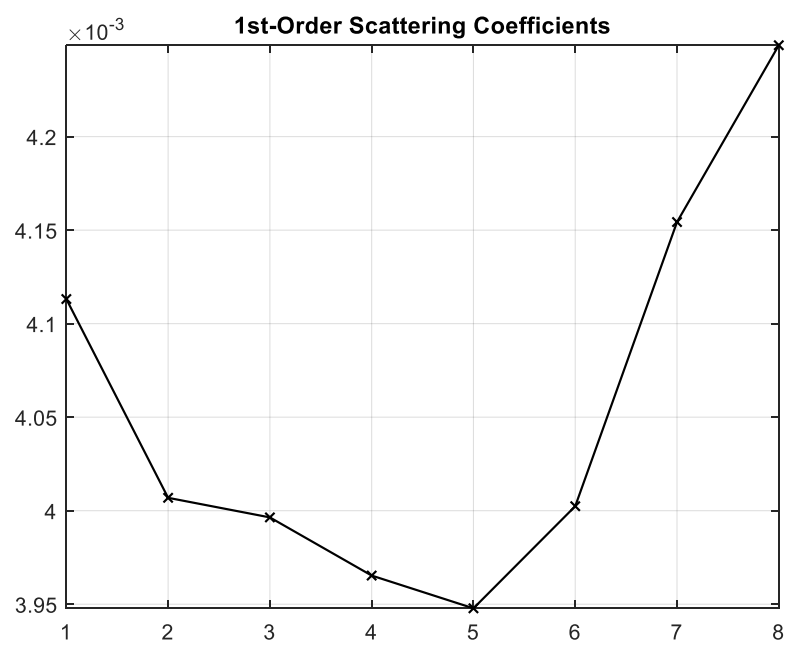

Figure 20: $1^{\text {st }}$ Order Scattering Coefficients

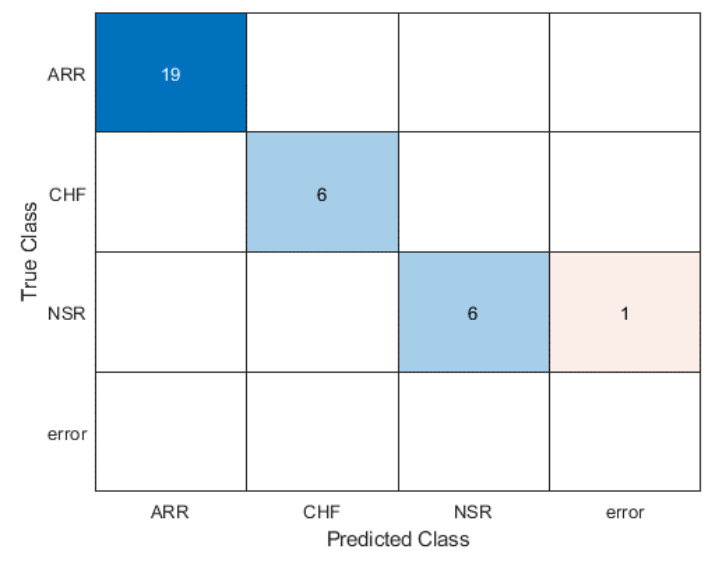

Figure 21: Confusion Matrix 


\section{Autoencoders}

Another important contribution of this resea rch work is a real-time implementation of a framework, designed on the basis of LabView and Python as an add-on which produces a real time powerful signal anomaly detection system. LabView 2020 Community edition is used for implementation of Python code with Python 3.6 version. In real time scenario, an Auto encoder based deep neural network is implemented to detect the a nomaly of acquired ECG signals. The model is implemented in keras and the model summary is shown in figure 22.

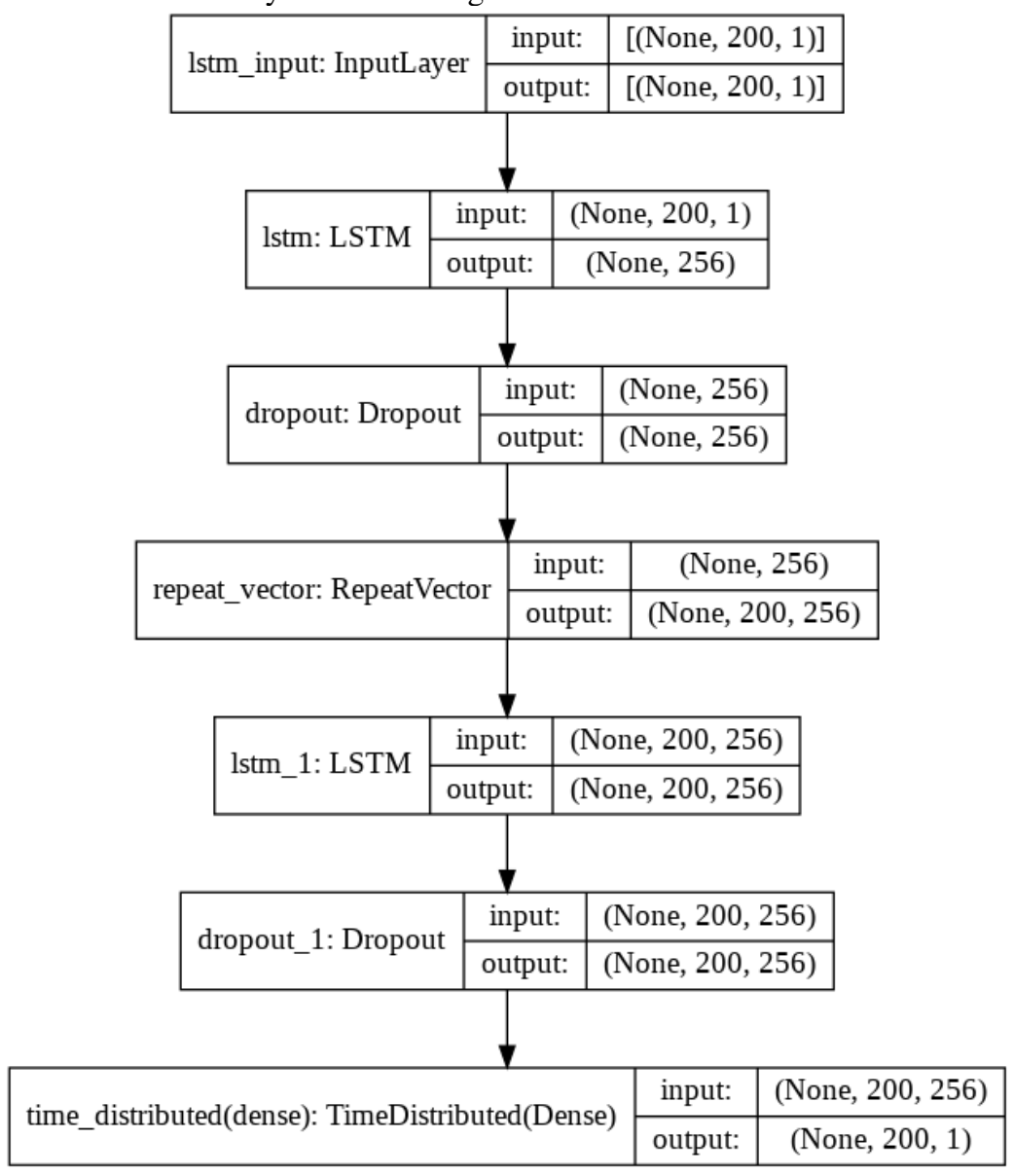

Figure 22: Model Summary

The model used in the classifier has simple structure of one LSTM layer with 256 followed by dropout layer with rate of 0.2 . Then repeat vector followed by decoder level of LSTM layer with 256 and Timedistrubuted layer is applied. Adam optimizer is used with "mae" loss. The average mae loss of 0.0072 for normal signals is achieved and 0.078 is achieved for anomaly signals. Fig 23 shows the distribution of normal signal mae loss while fig 24 shows the distribution of anomaly loss. 


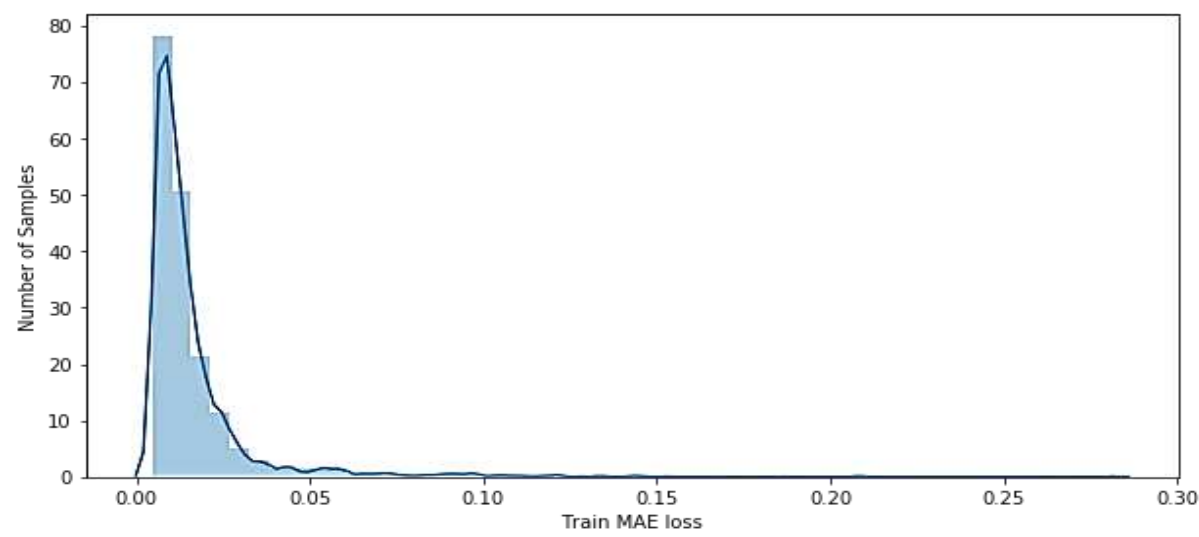

Figure 23: Distribution of Normal signals Loss Distribution of anomaly Loss

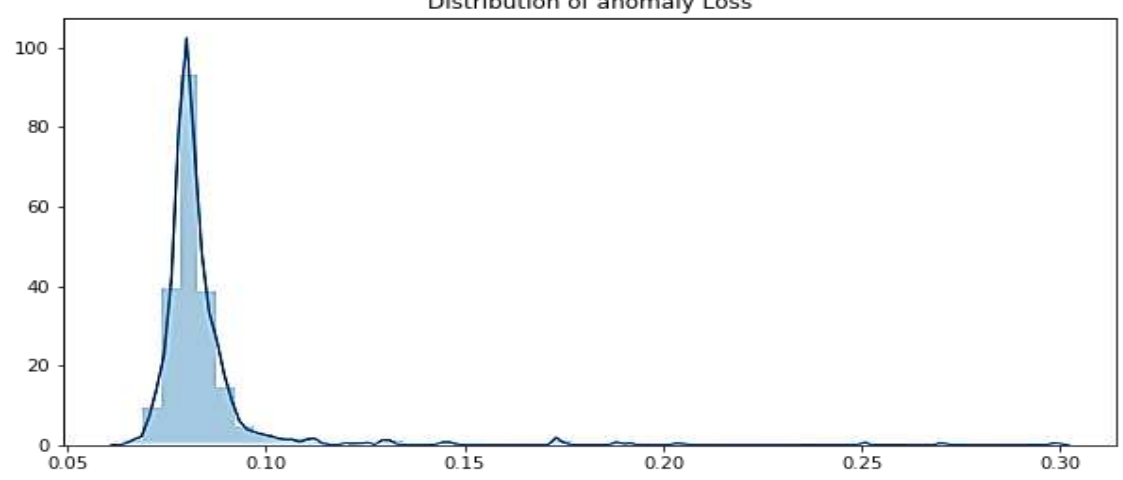

Figure 24:Distribution of Anomaly signals Loss

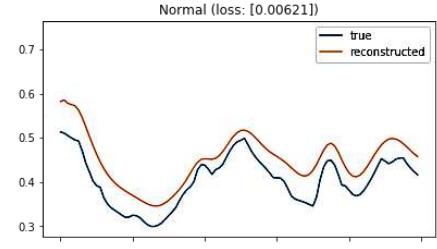

Anomaly (loss: [0.081])

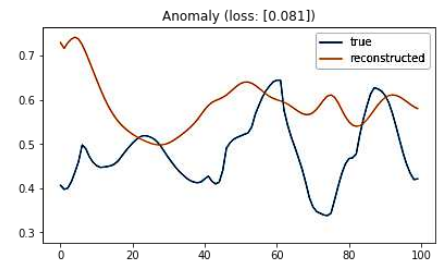

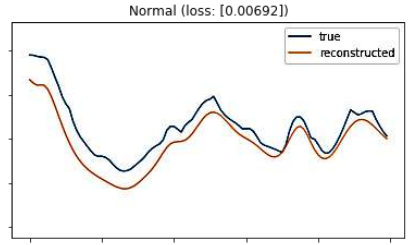

Anomaly (loss: [0.079])

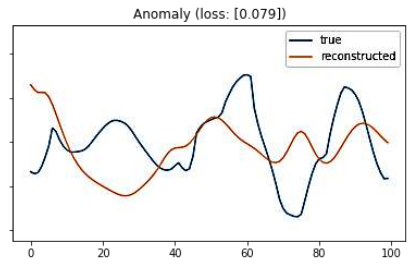

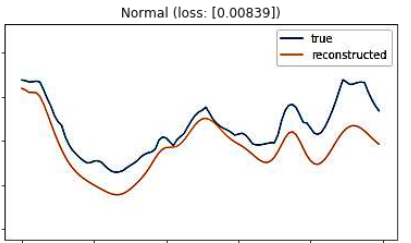

Anomaly (loss: [0.081])

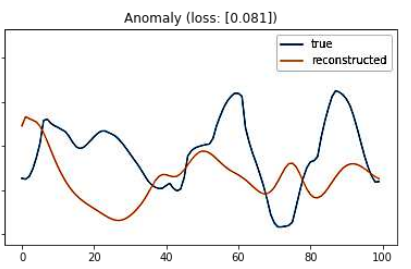

Figure 25: Reconstruction error of Normal \& Anomaly Signals

\section{Conclusion}

An IoT based healthcare framework has been designed and implemented for in time awareness of decline in physiological parameters, ultimately preventing probable loss. This system helps in improving medical services by providing a smart system capable of tracking and displaying the analysed state of patient's health using graphical representation. This information helps the doctor understand and prescribe some suitable medical treatment for immediate action.

One of the contributions in this research is extensive signal processing of acquired data from bio signal a cquisition framework, in order to understand the patient's condition. Analysis has been done by detecting PR, QRS, ST intervals, R-peaks in ECG signal, Heart Rate Variance, and standard deviation (SD) and variance measures, the median power frequency, and the slope of the Median Power Frequency in EMG signal. In the extended part of the research work of the proposed designed framework online signal anomaly detection is implemented using autoencoder. By setting 
reconstructed error thresholds of 0.02 in term of MAE loss, $98 \%$ accuracy is achieved for anomaly detection. If anomaly is detected then an intensive wavelet time scattering based featured are extracted and classified by multicla ss SVM with accuracy of $98.78 \%$ of 3 class ECG signals.

\section{DECLARATION:}

We declare that we will make readily reproducible materials described in the manuscript, including new software, databases and all relevant raw data, freely available to any scientist wishing to use them, without breaching participant confidentiality.

*Funding: NA

*Conflicts of interest/Competing interests: We have no conflicts of interest to disclose.

* Availability of data and material: Three datasets are used in this research including MIT-BIH Arrhythmia Database, MIT-BIH Normal Sinus Rhythm Database and The BIDMC Congestive Heart Failure Database.

*Code a vailability: NA

* Authors' contributions: One of the contributions in this research is extensive signal processing of a cquired da ta from bio signal acquisition framework, in order to understand the patient's condition. Analysis has been done by detecting PR, QRS, ST intervals, R-peaks in ECG signal, Heart Rate Variance, and standard deviation (SD) and variance measures, the median power frequency, and the slope of the Median Power Frequency in EMG signal. In the extended part of the research work of the proposed designed framework online signal anomaly detection is implemented using autoencoder. By setting reconstructed error thresholds of 0.02 in term of MAE loss, $98 \%$ accuracy is achieved for anomaly detection. If anomaly is detected then an intensive wavelet time scattering based featured are extracted and classified by multiclass SVM with accuracy of $98.78 \%$ of 3 class ECG signals.

\section{References}

1. Faust, O., Hagiwara, Y., Hong, T. J., Lih, O. S., \& Acharya, U. R. (2018). Deep learning for healthcare applications based on physiological signals: A review. Computer Methods and Programs in Biomedicine, 161,1-13. https://doi.org/10.1016/j.cmpb.2018.04.005

2. Yeasmin, S. (2019). Benefits of Artificial Intelligence in Medicine. 2nd International Conference on Computer Applications and Information Security, ICCAIS 2019, 1-6. https://doi.org/10.1109/CAIS.2019.8769557

3. Chauhan, S., \& Vig, L. (2015). Anomaly detection in ECG time signals via deep long shortterm memory networks. Proceedings of the 2015 IEEE International Conference on Data Science and Advanced Analytics, DSAA 2015, (October). https://doi.org/10.1109/DSAA.2015.7344872

4. Nawaz, M., Ahmed, J., Abbas, G., \& Ur Rehman, M. (2021). Signal Analysis and Anomaly Detection of IoT-Based Healthcare Framework, 1-6. https://doi.org/10.1109/gcwot49901.2020.9391621

5. Tyagi, S., Agarwal, A., \& Maheshwari, P. (2016). A conceptual framework for IoT-based healthcare system using cloud computing. Proceedings of the 2016 6th International Conference - Cloud System and Big Data Engineering, Confluence 2016, (January), 503507. https://doi.org/10.1109/CONFLUENCE.2016.7508172

6. Al-Emran, M., Malik, S. I., \& Al-Kabi, M. N. (2020). A Survey of Internet of Things (IoT) in Education: Opportunities and Challenges (pp. 197-209). https://doi.org/10.1007/978-3030-24513-9_12

7. Shin, D., Kim, S., Yeom, S., Kwon, O. J., \& Shin, D. (2018). Ubiquitous healthcare system for analysis of chronic patients' biological and lifelog data. IEEE Access, 6(c), 8909-8915. https://doi.org/10.1109/ACCESS.2018.2805304

8. Catarinucci, L., de Donno, D., Mainetti, L., Palano, L., Patrono, L., Stefanizzi, M. L., \& Tarricone, L. (2015). An IoT-Aware Architecture for Smart Healthcare Systems. IEEE Internet of Things Journal,2(6), 515-526.https://doi.org/10.1109/JIOT.2015.2417684

9. Acharya, U. R., Oh, S. L., Hagiwara, Y., Tan, J. H., Adam, M., Gertych, A., \& Tan, R. S. (2017). A deep convolutional neural network model to classify heartbeats. Computers in Biology and Medicine, 89, 389-396.https://doi.org/10.1016/j.compbiomed.2017.08.022

10. Zhao, B., Lu, H., Chen, S., Liu, J., \& Wu, D. (2017). Convolutionalneural networks for time 
series classification. Journal of Systems Engineering and Electronics, 28(1), 162-169. https://doi.org/10.21629/JSEE.2017.01.18

11. Ismail Fawaz, H., Forestier, G., Weber, J., Idoumghar, L., \& Muller, P.-A. (2019). Deep learning for time series classification: a review. Data Mining and Knowledge Discovery, 33(4), 917-963. https://doi.org/10.1007/s10618-019-00619-1

12. Mahmud, M. A., Abdelgawad, A., Yelamarthi, K., \& Ismail, Y. A. (2017). Signal processing techniques for IoT-based structural health monitoring. In 2017 29th International Conference on Microelectronics (ICM) (pp. 1-5). IEEE. https://doi.org/10.1109/ICM.2017.8268825

13. Azariadi, D., Tsoutsouras, V., Xydis, S., \& Soudris, D. (2016). ECG signal analysis and arrhythmia detection on IoT wearable medical devices. In 20165 th International Conference on Modern Circuits and Systems Technologies (MOCAST) (pp. 1-4). IEEE. https://doi.org/10.1109/MOCAST.2016.7495143

14. Wang, Y., Kung, L., \& Byrd, T. A. (2018). Big data analytics: Understanding its capabilities and potential benefits for healthcare organizations. Technological Forecasting and Social Change, 126, 3-13.https://doi.org/10.1016/j.techfore.2015.12.019

15. Kim, S., Yeom, S., Kwon, O.-J., Shin, D., \& Shin, D. (2018). Ubiquitous Healthcare System for Analysis of Chronic Patients' Biological and Lifelog Data. IEEE Access, 6, 8909-8915. https://doi.org/10.1109/ACCESS.2018.2805304

16. Shin, D., Shin, D., \& Shin, D. (2016). Ubiquitous Healthcare Platform for Chronic Patients. In 2016 International Conference on Platform Technology and Service (PlatCon) (pp. 1-6). IEEE. https://doi.org/10.1109/PlatCon.2016.7456836

17. Debayle, J., Hatami, N., \& Gavet, Y. (2018). Classification of time-series images using deep convolutional neural networks. In J. Zhou, P. Radeva, D. Nikolaev, \& A. Verikas (Eds.), Tenth International Conference on Machine Vision (ICMV 2017) (p. 23). SPIE. https://doi.org/10.1117/12.2309486

18. Geurts, P. (2001). Pattern Extraction for Time Series Classification (pp. 115-127). https://doi.org/10.1007/3-540-44794-6_10

19. Karim, F., Majumdar, S., Darabi, H., \& Harford, S. (2019). Multivariate LSTM-FCNs for time series classification. Neural Networks, 116, 237-245. https://doi.org/10.1016/j.neunet.2019.04.014

20. Kate, R. J. (2016). Using dynamic time warping distances as features for improved time series classification. Data Mining and Knowledge Discovery, 30(2), 283-312. https://doi.org/10.1007/s10618-015-0418-x

21. U. R. Acharya, H. Fujita, S. L. Oh, Y. Hagiwara, J. H. Tan, M. A. (n.d.). Application of deep convolutional neural network for automated detection of myocardial infarction using ecg signals,. Information Sciences 415, 190-198.

22. Chen, Z., Yeo, C. K., Lee, B. S., \& Lau, C. T. (2018). Autoencoder-based network anomaly detection. In 2018 Wireless Telecommunications Symposium (WTS) (pp. 1-5). IEEE. https://doi.org/10.1109/WTS.2018.8363930

23. Sugimoto, K., Kon, Y., Lee, S., \& Okada, Y. (2019). Detection and localization of myocardial infarction based on a convolutional autoencoder. Knowledge-Based Systems, 178, 123-131.https://doi.org/10.1016/j.knosys.2019.04.023

24. Theopaga, A. K., Rizal, A., \& S. (2014). DESIGN AND IMPLEMENTATION OF PID CONTROL BASED BABY INCUBATOR. Journal of Theoretical \& Applied Information Technology.

25. Rajendra Acharya, U., Paul Joseph, K., Kannathal, N., Lim, C. M., \& Suri, J. S. (2006). Heart rate variability: a review. Medical \& Biological Engineering \& Computing, 44(12), 1031-1051.https://doi.org/10.1007/s11517-006-0119-0

26. Costa, M. V, Pereira, L. A., Oliveira, R. S., Pedro, R. E., Camata, T. V, Abrão, T., ... Altimari, L. R. (2010). Fourier and wavelet spectral analysis of EMG signals in maximal constant load dynamic exercise. In 2010 Annual International Conference of the IEEE Engineering in Medicine and Biology (pp. 4622-4625). IEEE. https://doi.org/10.1109/IEMBS.2010.5626474

27. Rhif, M., Ben Abbes, A., Farah, I., Martínez, B., \& Sang, Y. (2019). Wavelet Transform Application for/in Non-Stationary Time-Series Analysis: A Review. Applied Sciences, 9(7), 1345.https://doi.org/10.3390/app9071345

28. Mathswork. (n.d.). Retrieved from https://ww2.mathworks.cn/help/wavelet/ug/ecg-signal- 
classification-using-wavelet-time-scattering.html

29. Physionet.org. (n.d.). Retrieved from https://www.physionet.org/ 
Figures

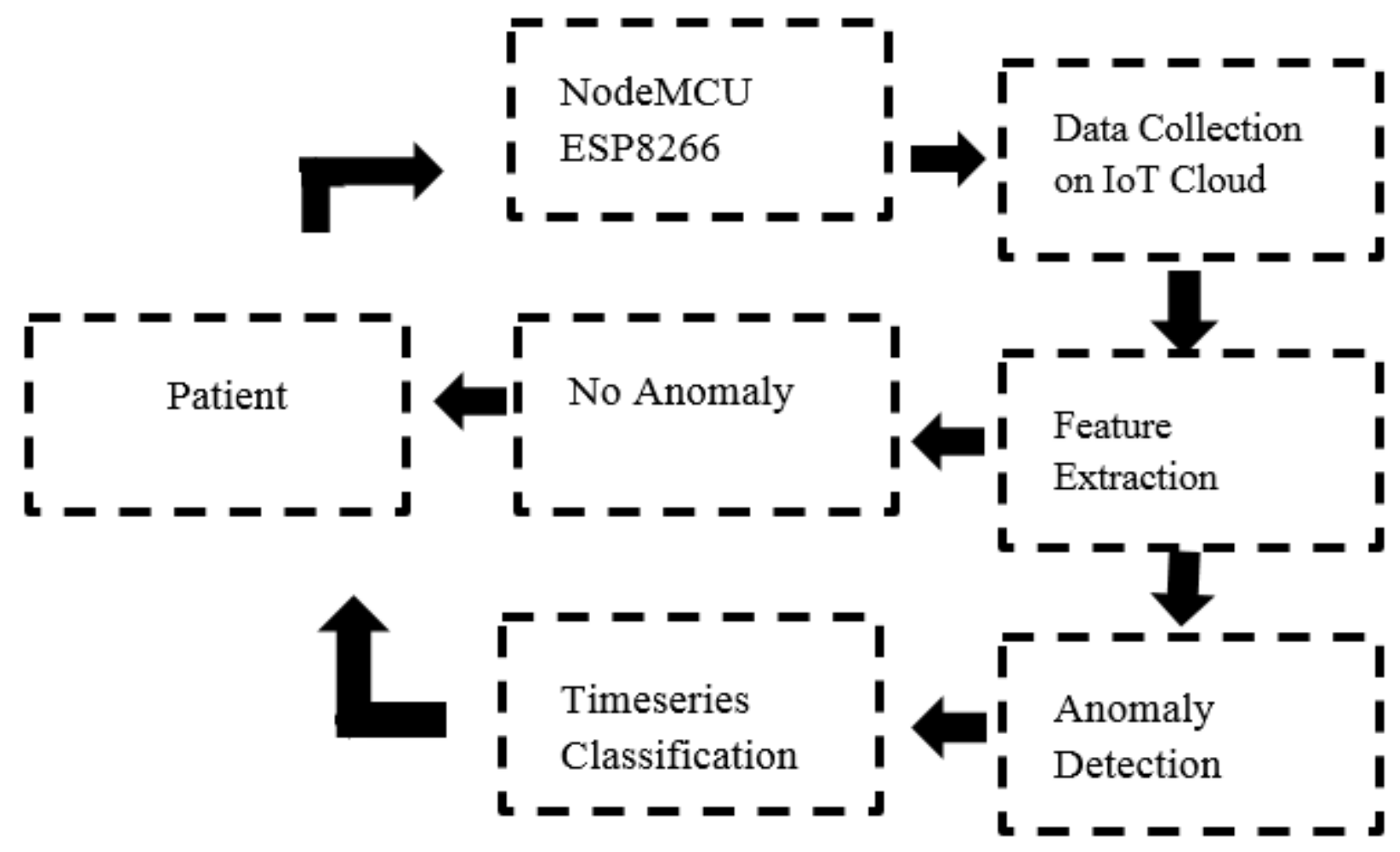

Figure 1

Block Diagram of loT-Based Healthcare System with Signal Processing and anomaly detection 


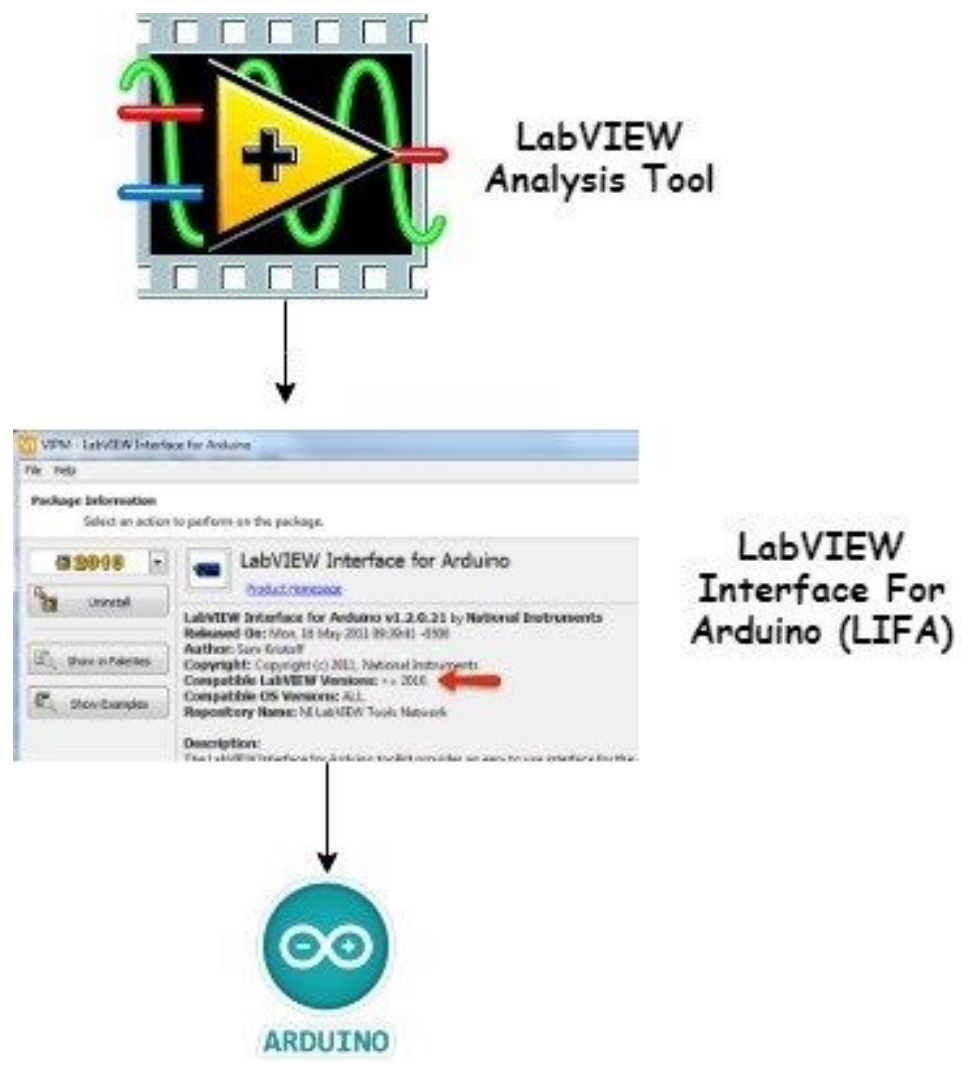

\section{Figure 2}

System Integration for Signal Analysis

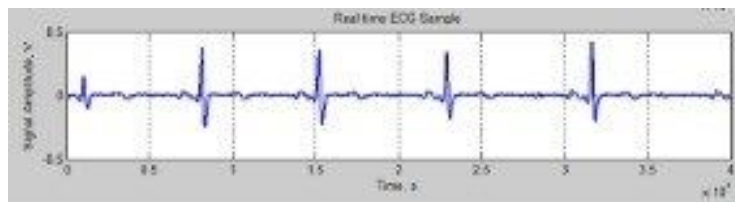

ECG Signal

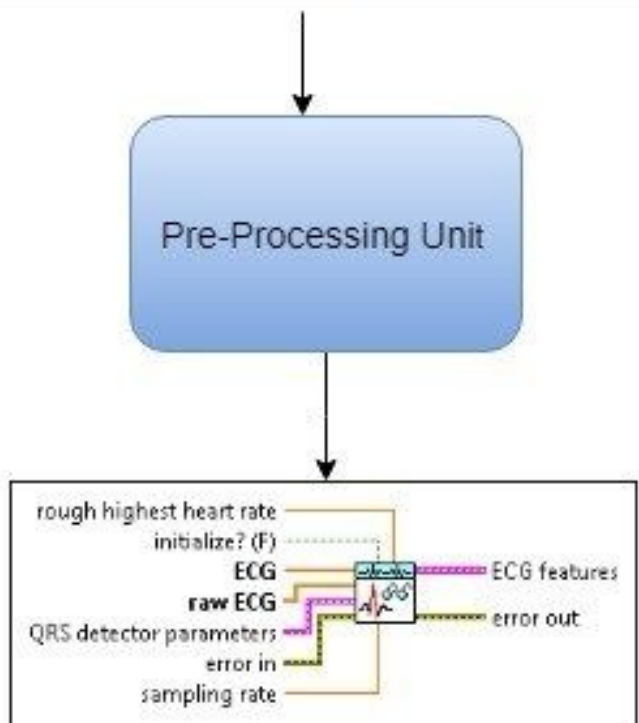

Feature

Extraction

\section{Figure 3}

Signal processing steps in ECG analysis 

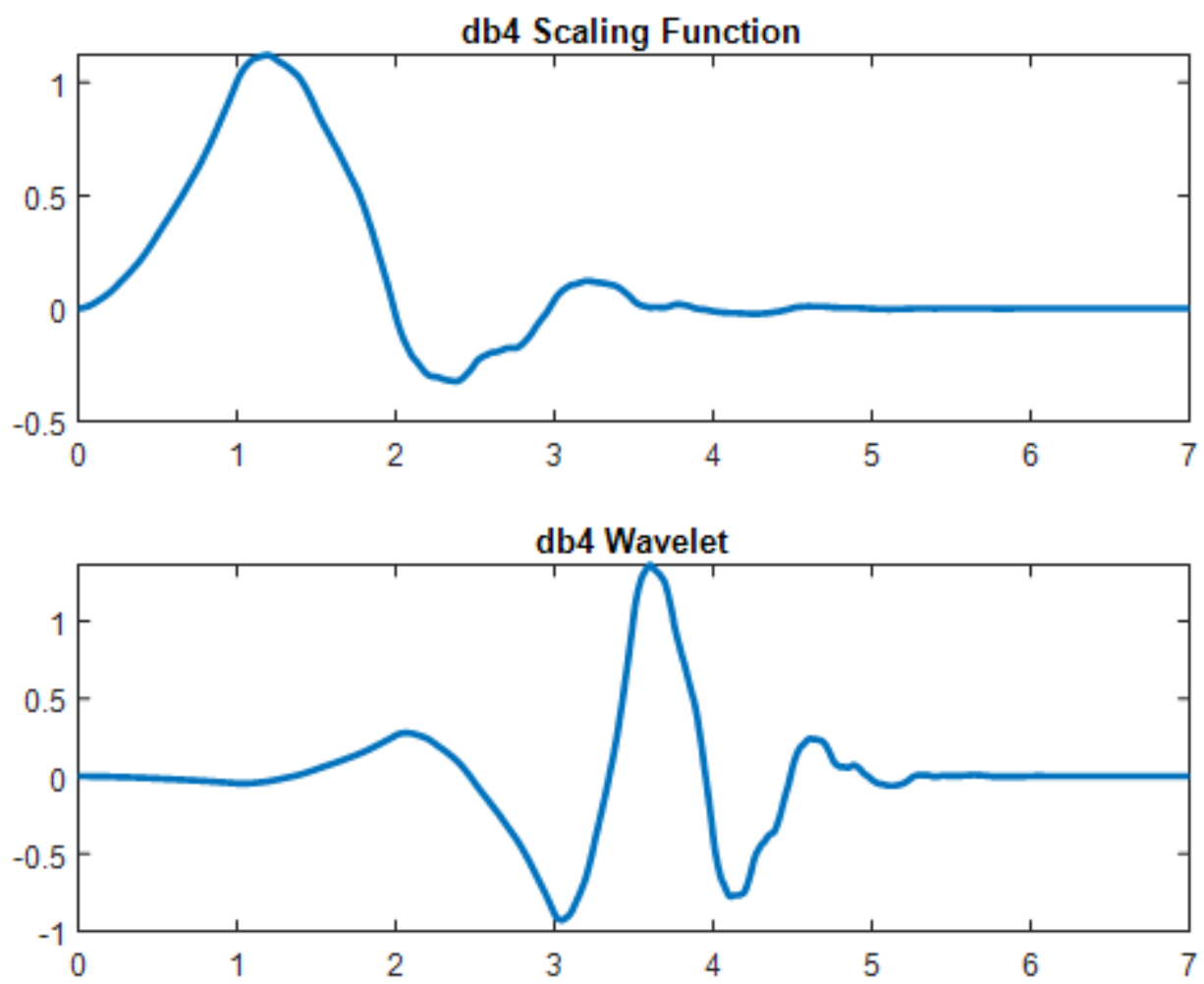

Figure 4

Wavelet Transform and Scaling Function
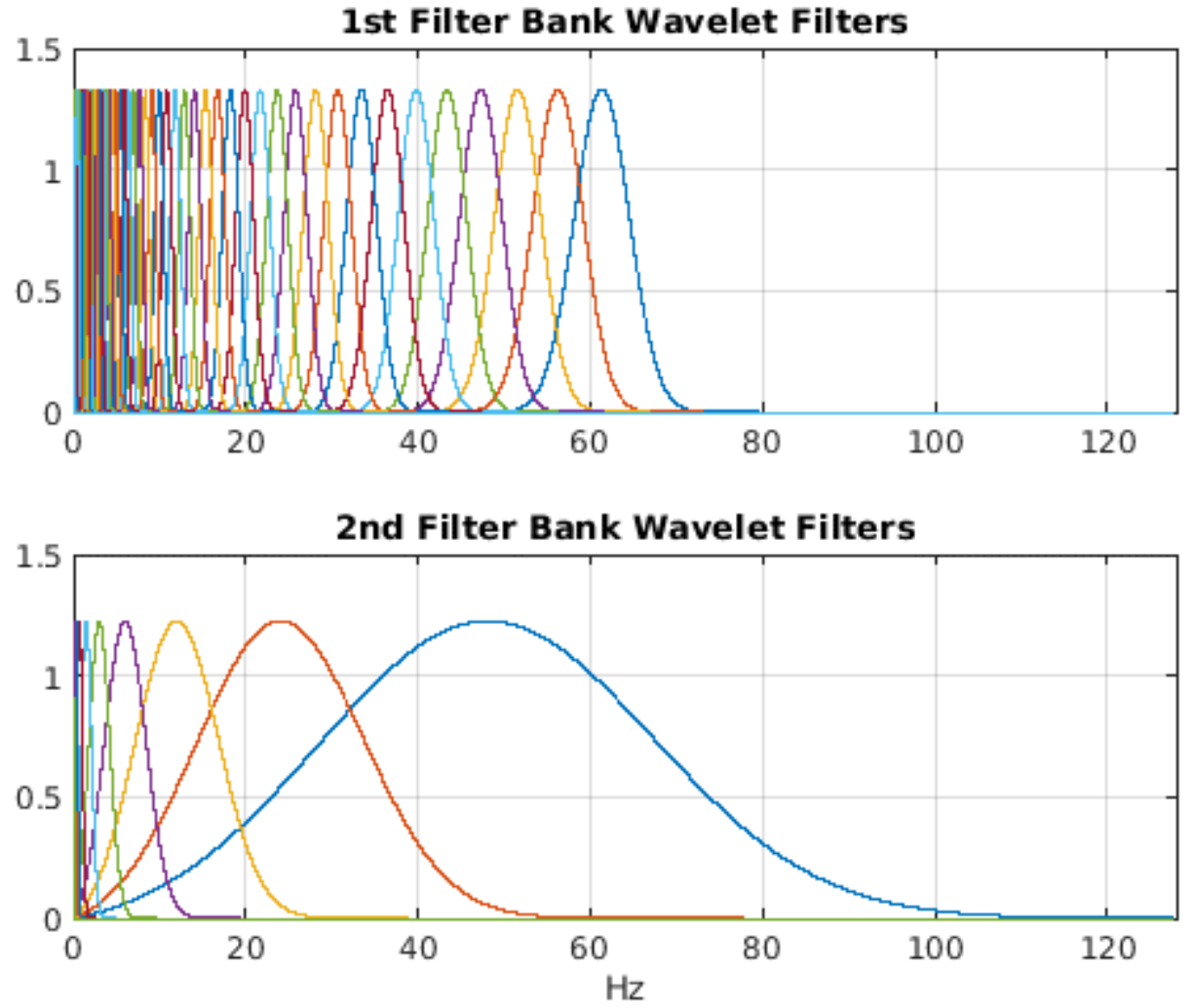
Figure 5

1st Filter and 2nd Filter Bank Wavelet Filters [28]

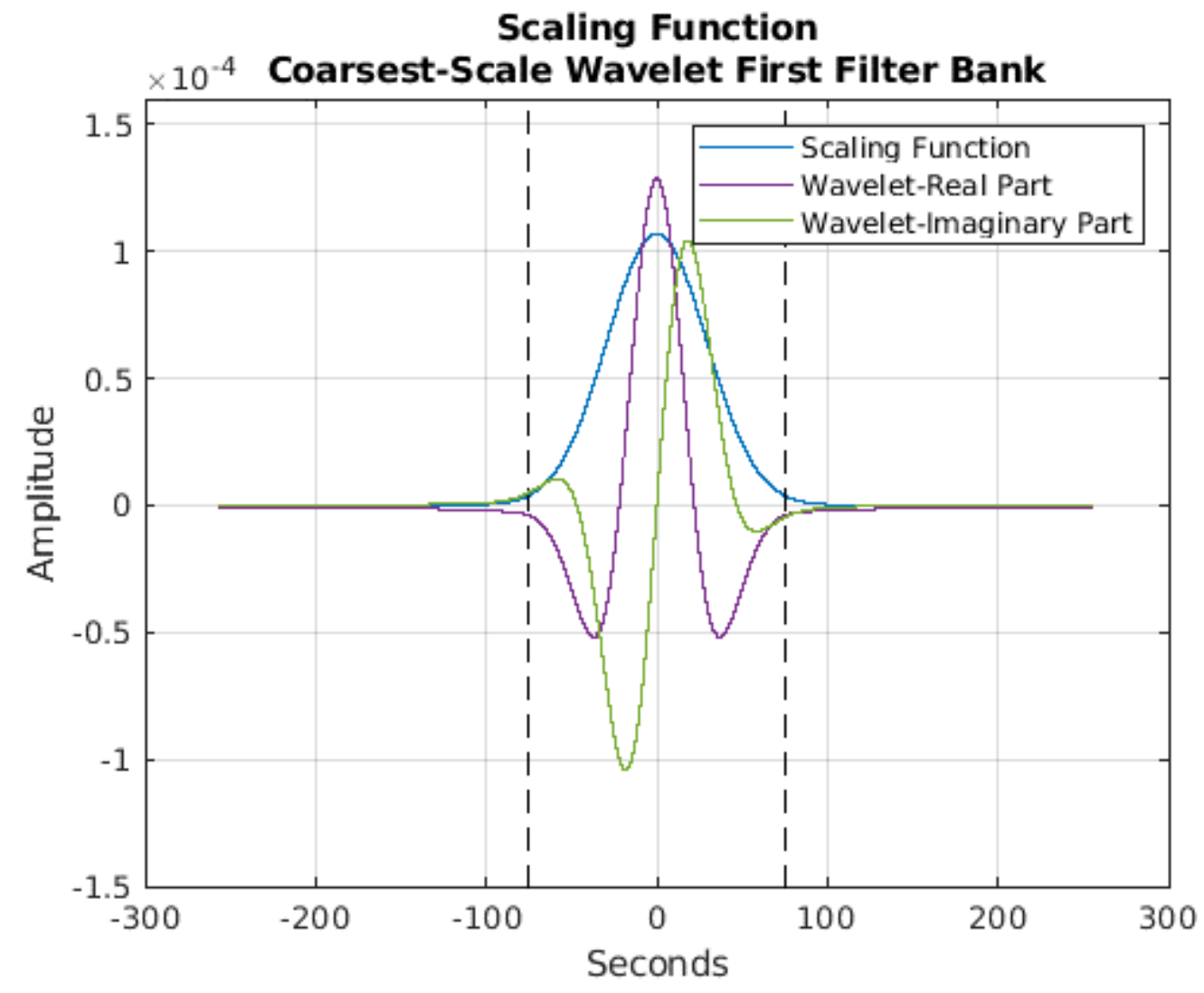

Figure 6

Coarsest-scale Wavelet First Filter Bank[28]

Data 2

\begin{tabular}{|l|l|}
\hline Plot 0 & $\triangle$ \\
\hline
\end{tabular}

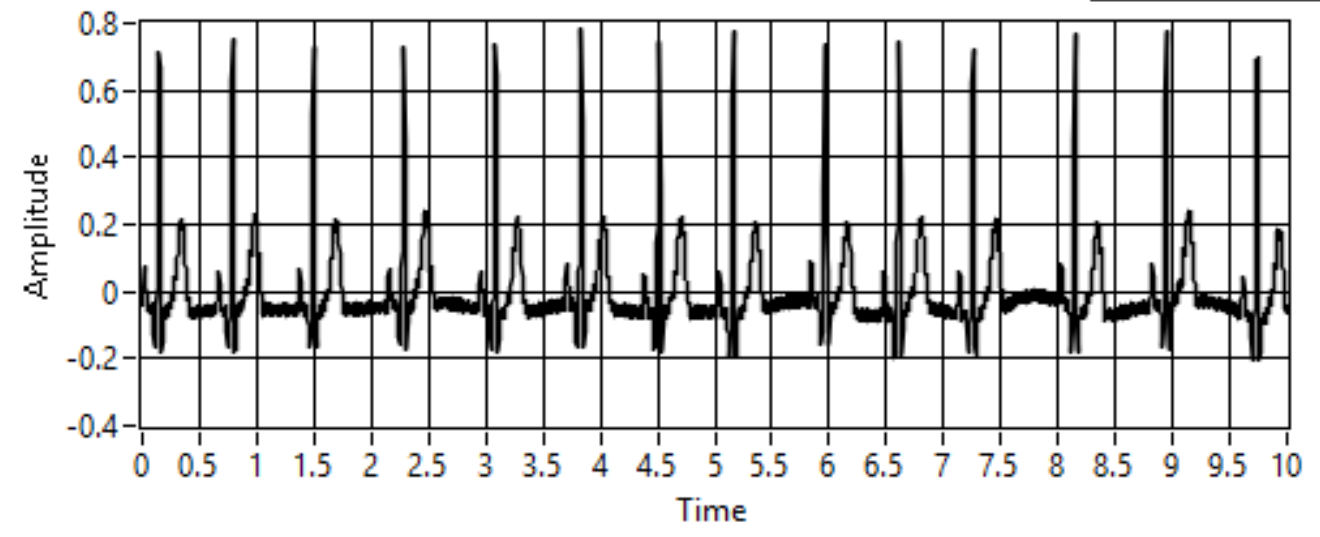

Figure 7

Unfiltered ECG Signal 
Data 3

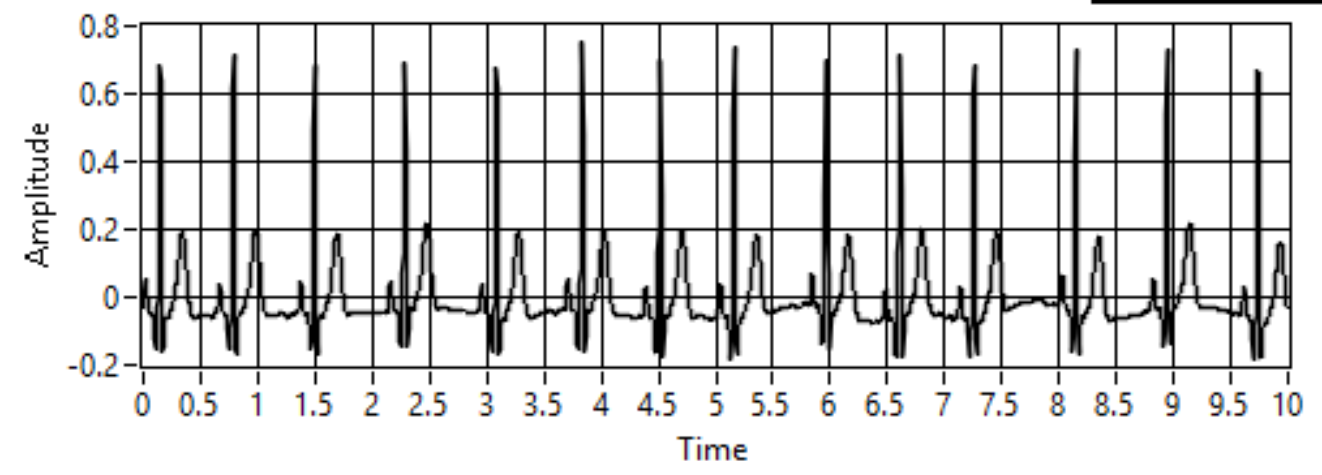

Figure 8

De-trend \& De-noised ECG signal

QRS Interval

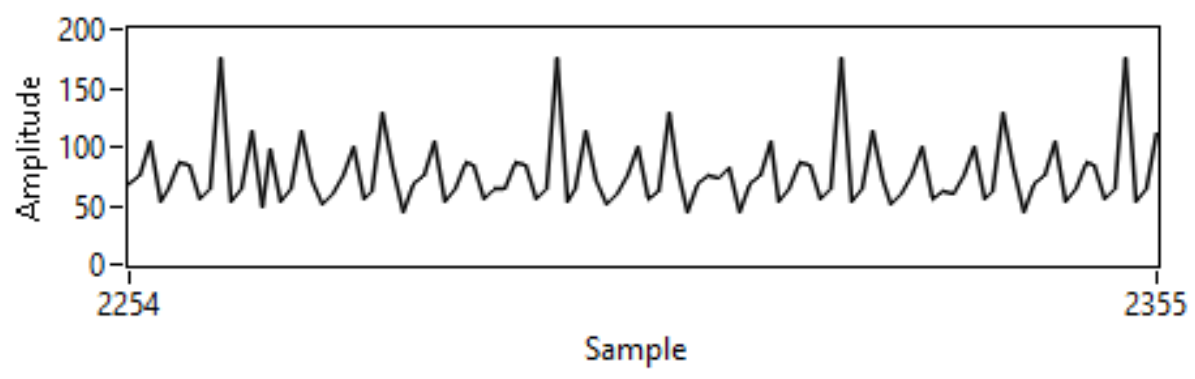

Figure 9

Peak and Interval detection in ECG Signal

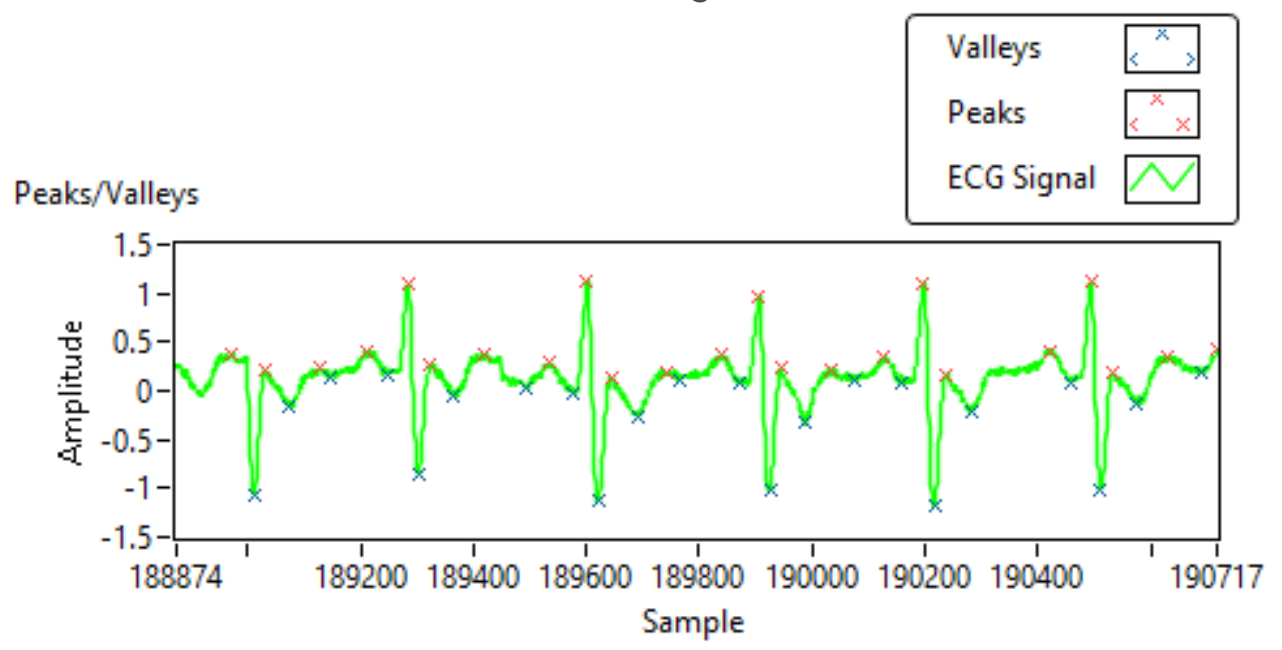

Figure 10

Detection of QRS interval 


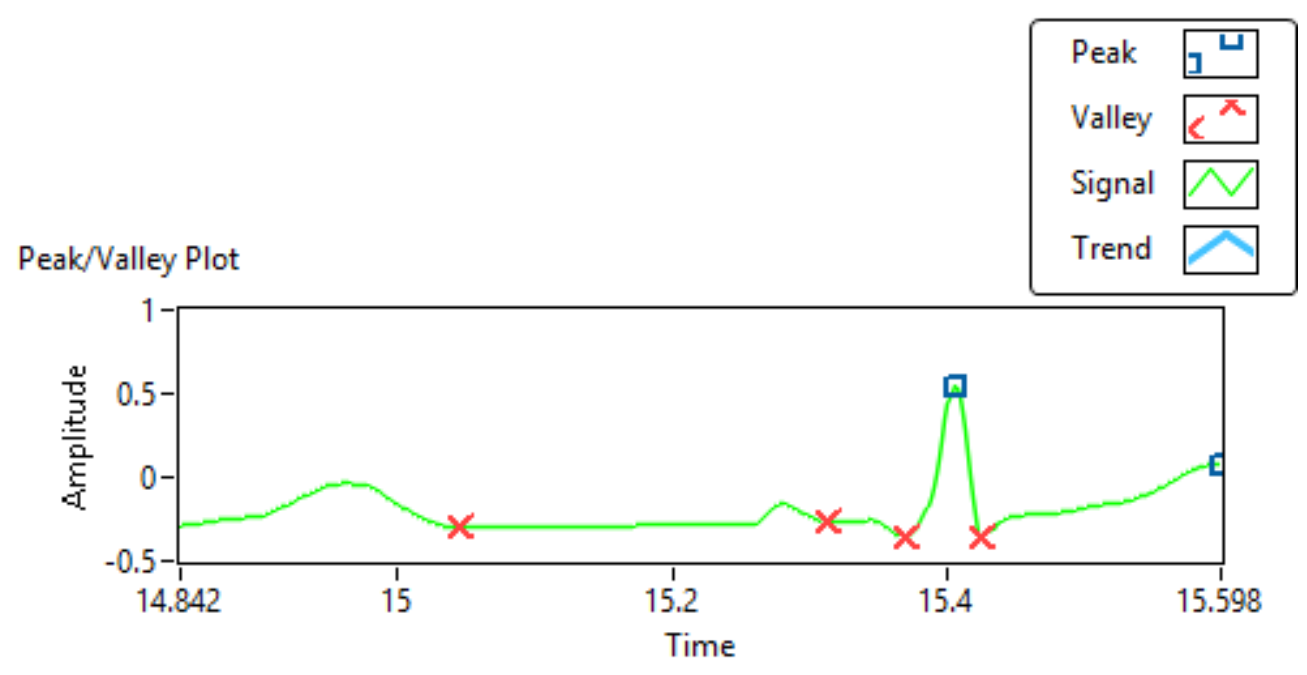

\section{Figure 11}

Peak/Interval detection in ECG Signal HRV

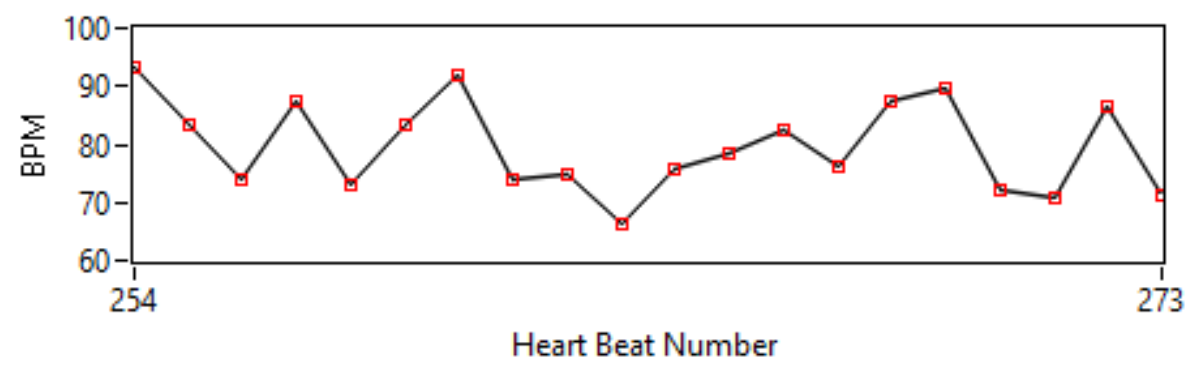

Figure 12

Measurement of HR

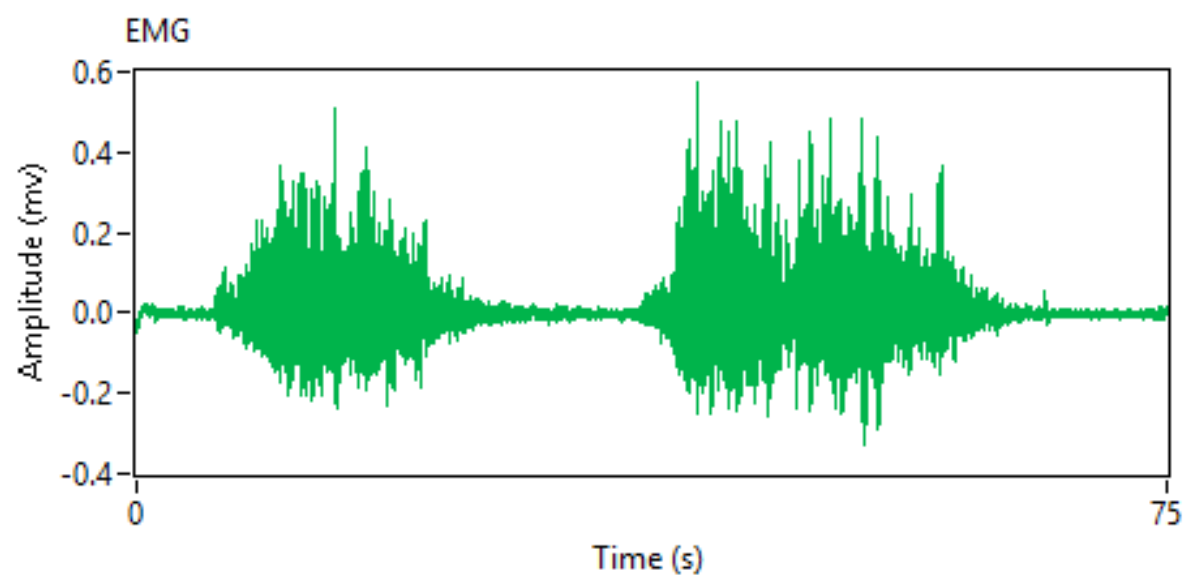

Figure 13

EMG Signal 


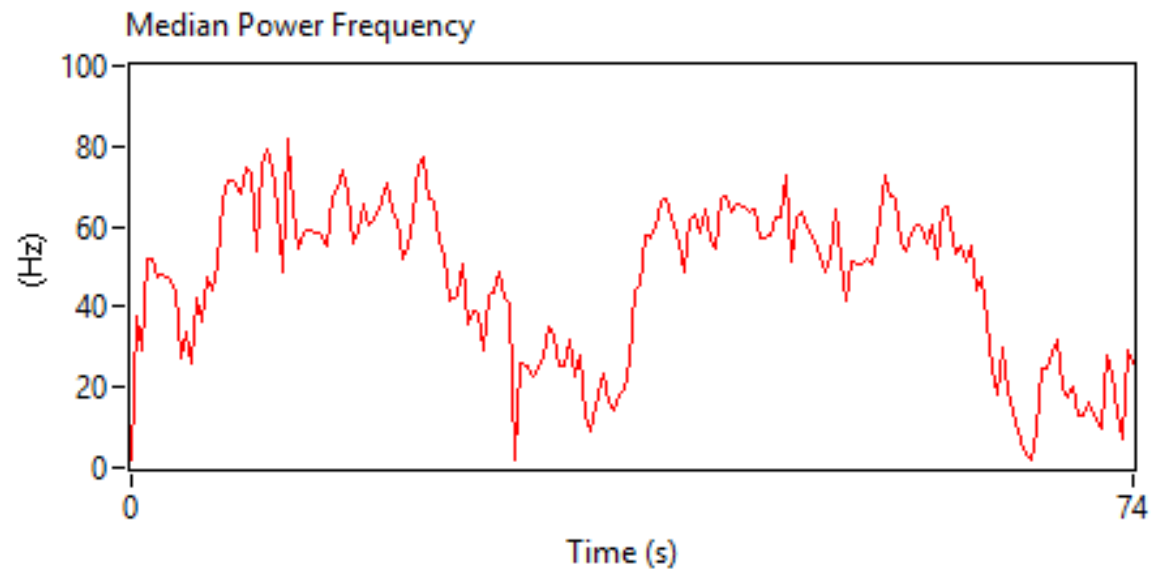

Figure 14

Median Power Frequency of EMG Signal

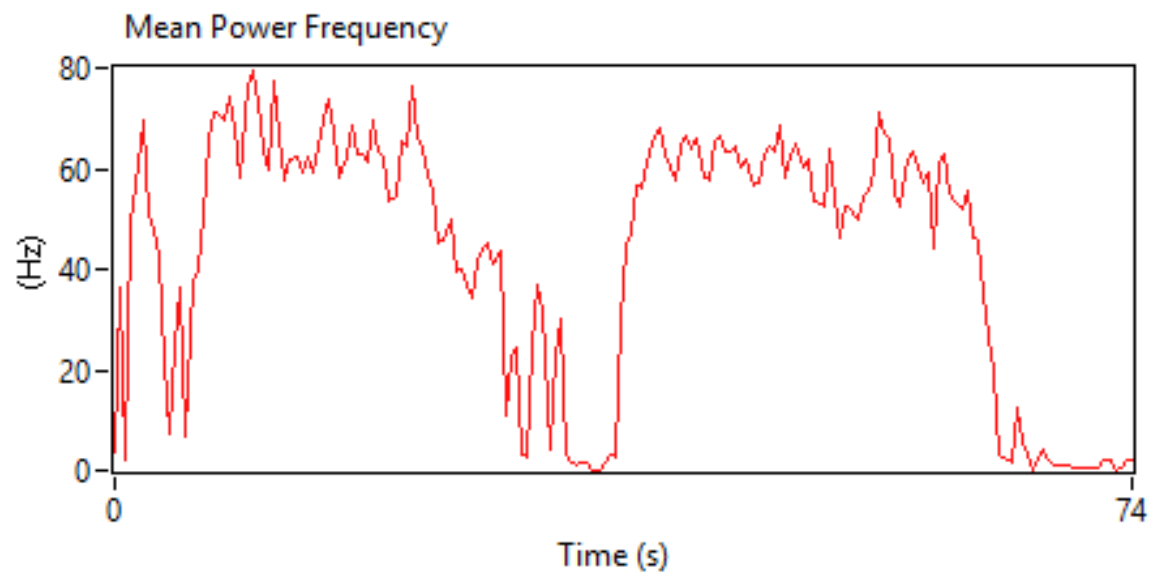

Figure 15

Mean Power Frequency of EMG Signal

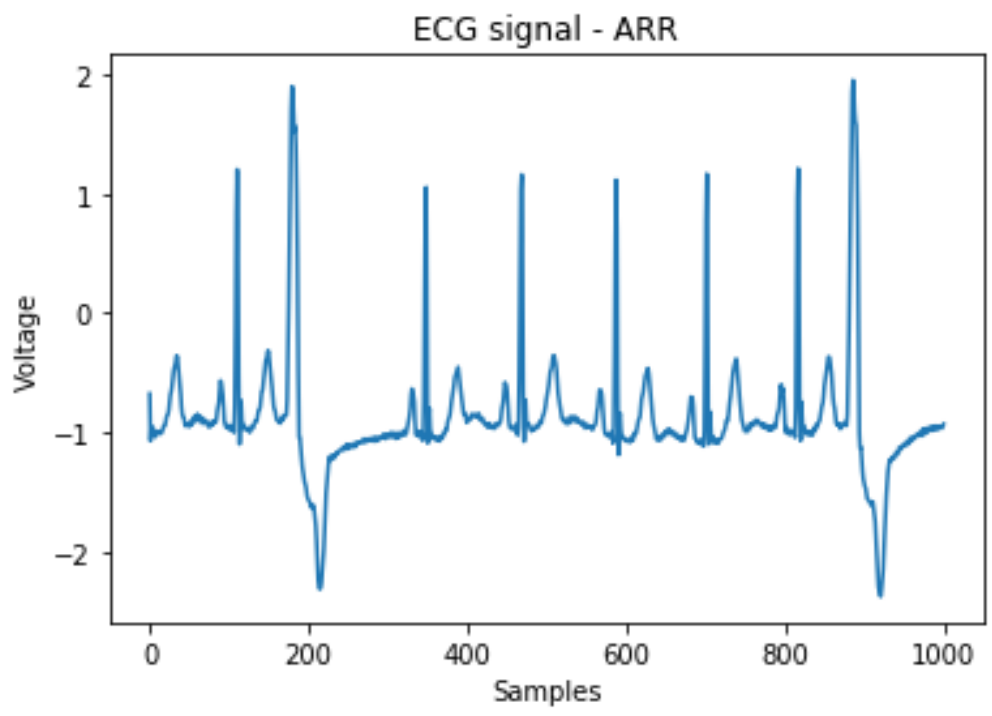

Figure 16 
ECG Signal for Arrythmia

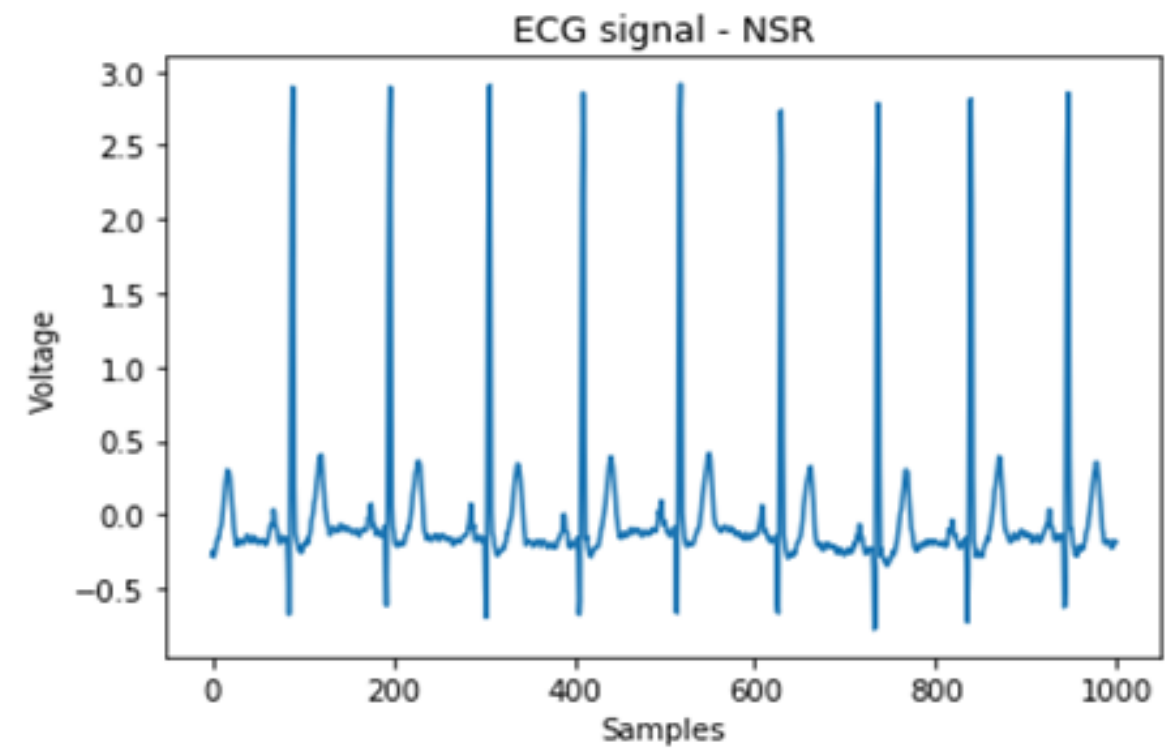

Figure 17

ECG Signal for Normal Sinus rhythm

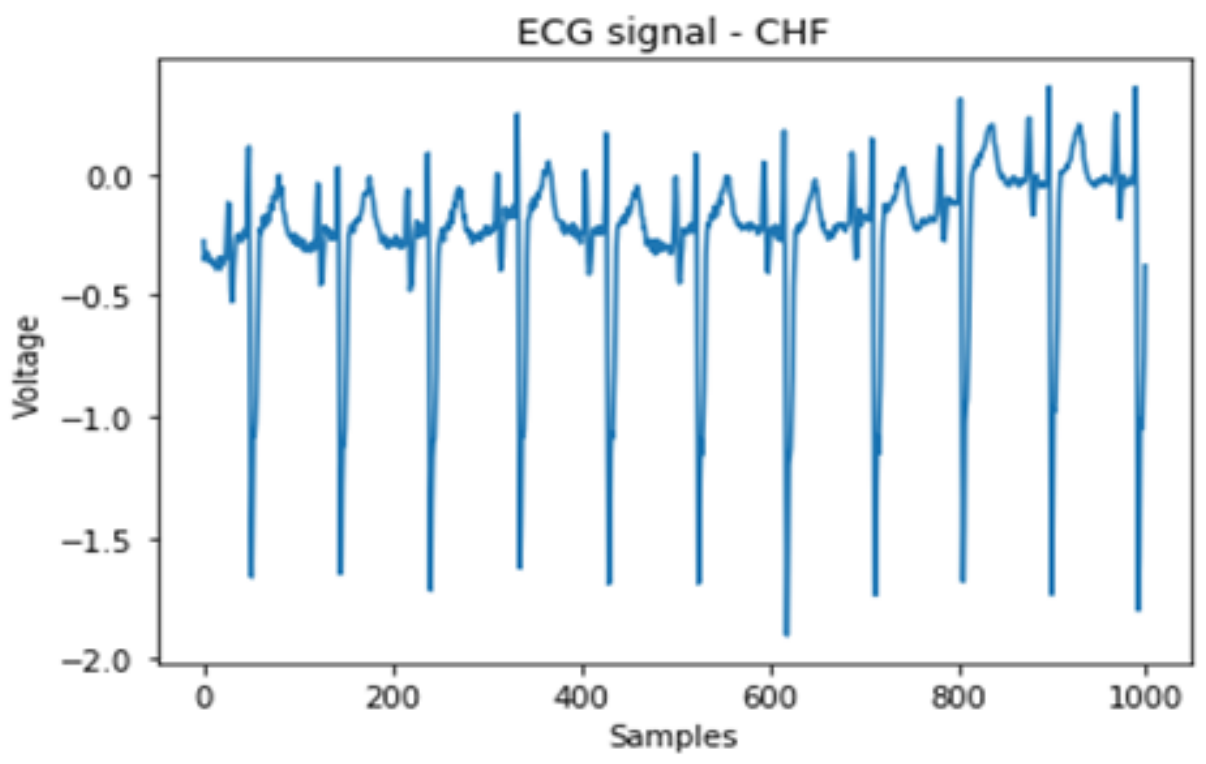

Figure 18

ECG Signal for Congestive Heart Failure 


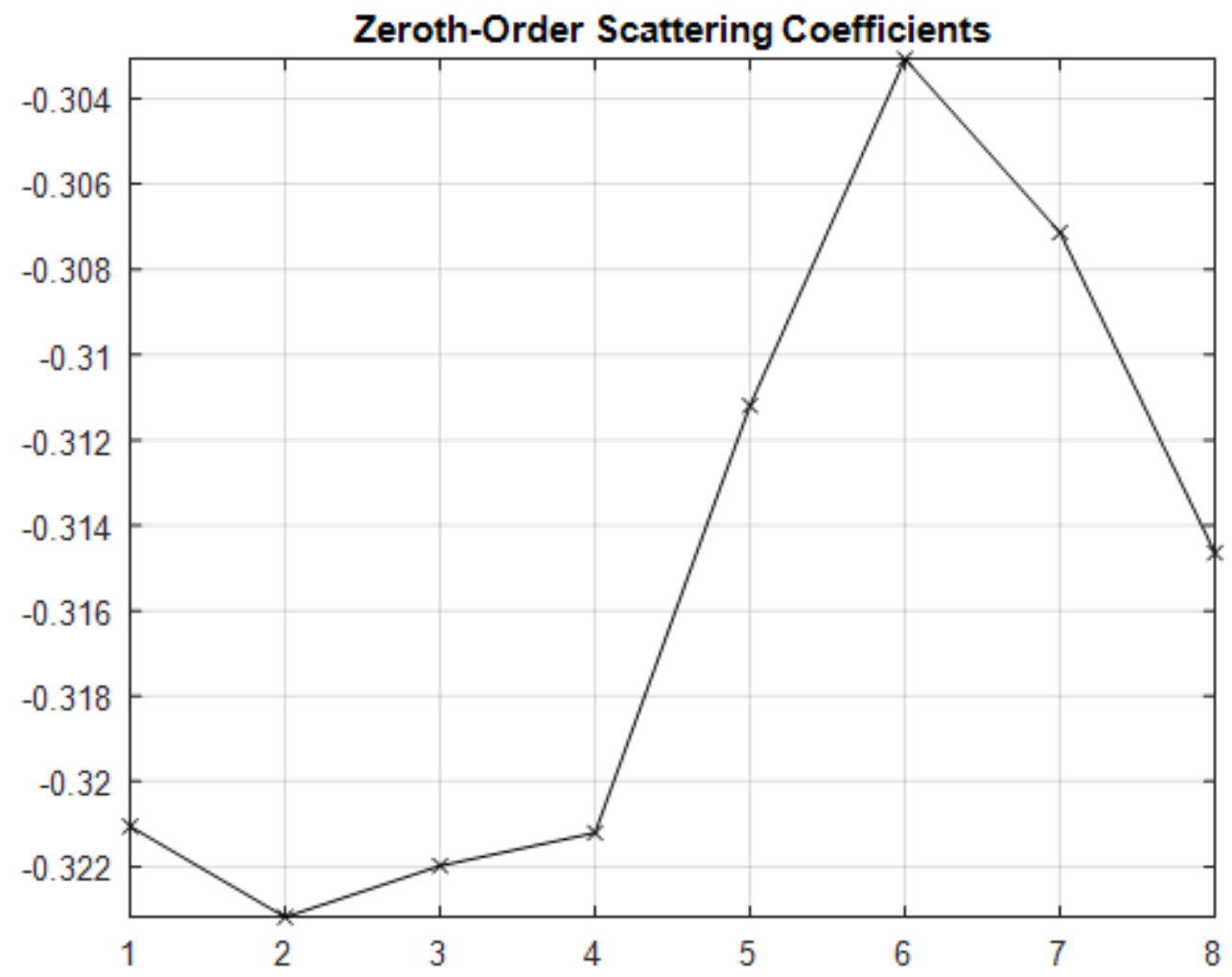

Figure 19

Zeroth-order Scattering Coefficients

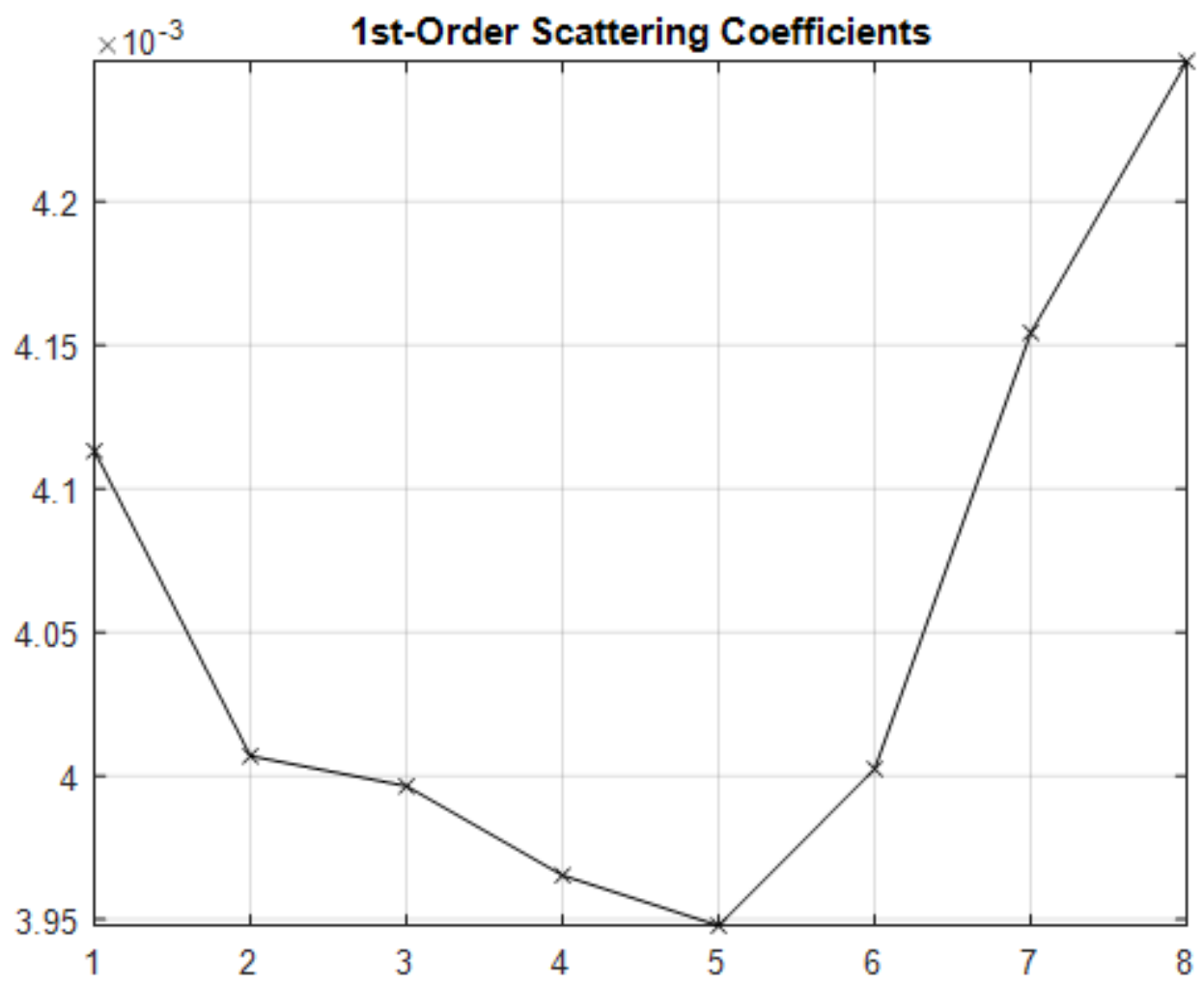


Figure 20

1st Order Scattering Coefficients

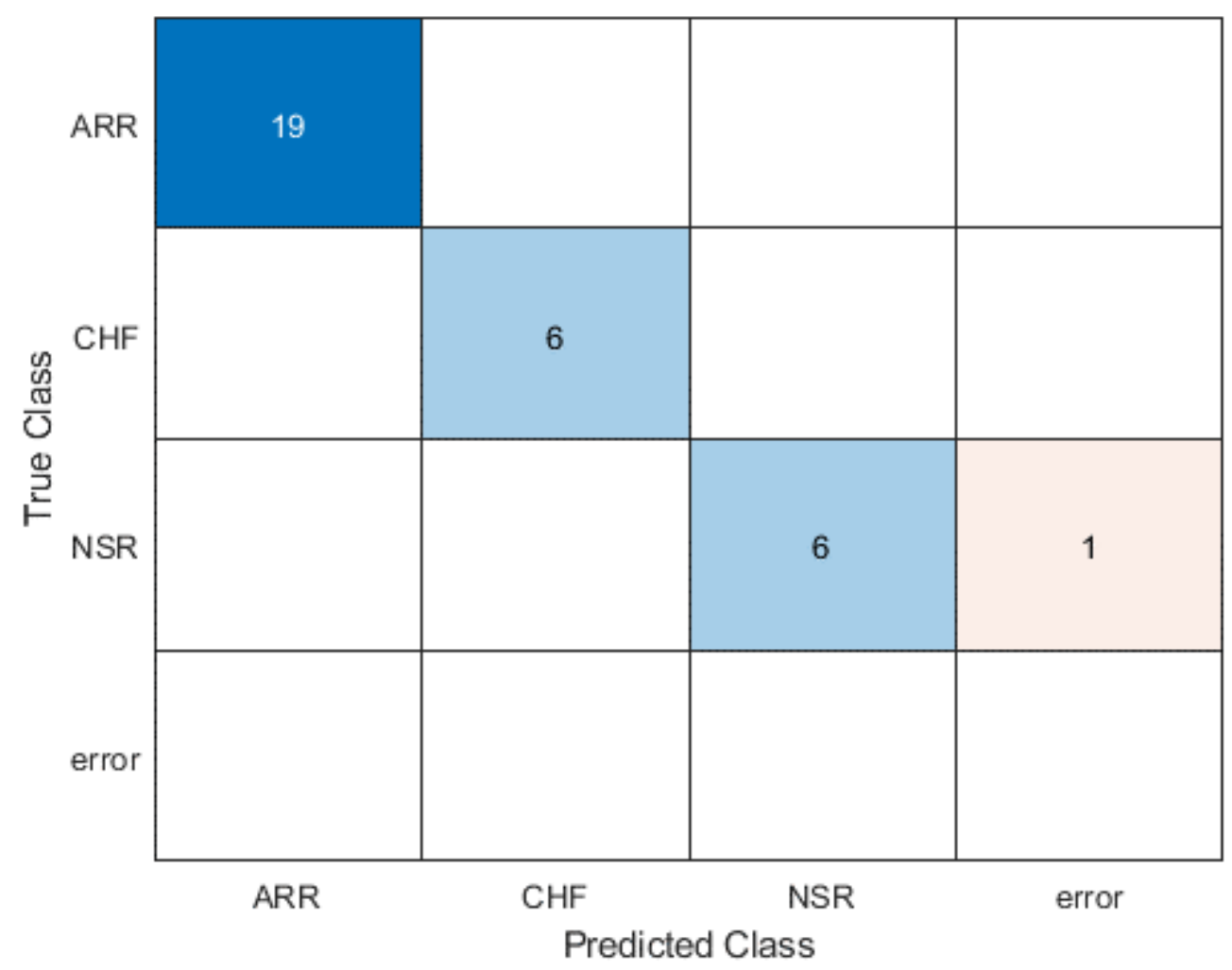

Figure 21

Confusion Matrix 


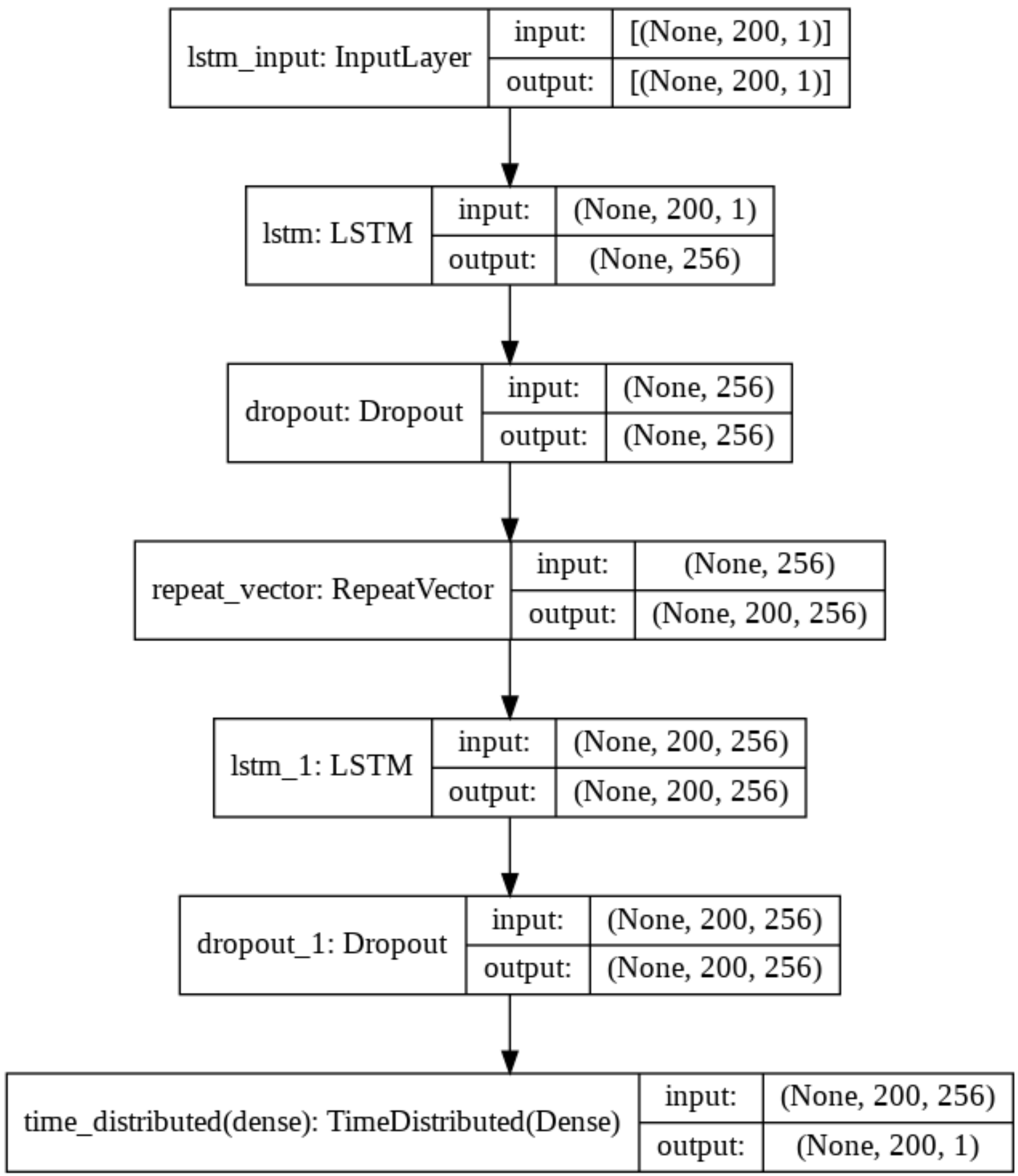

Figure 22

Model Summary 


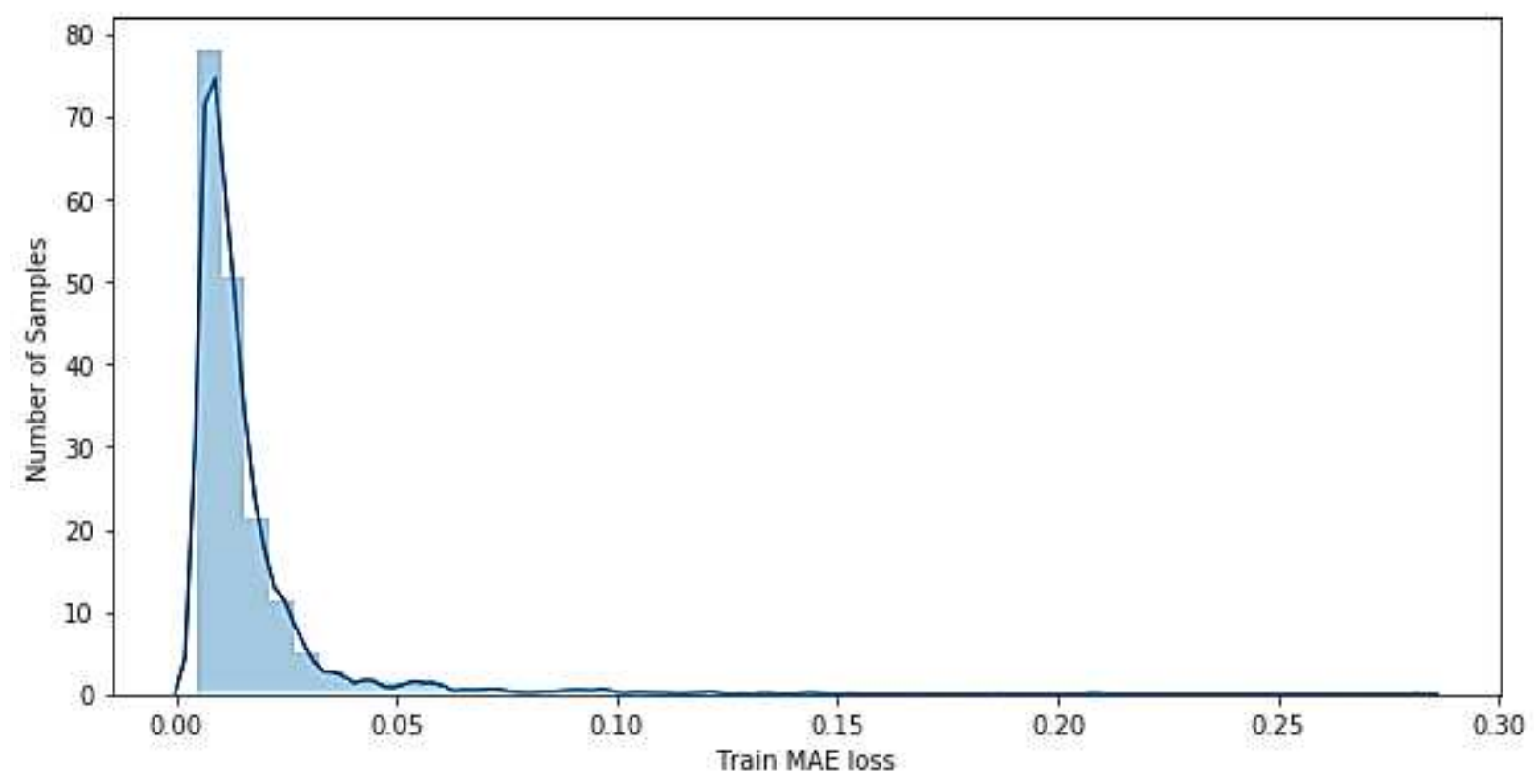

Figure 23

Distribution of Normal signals Loss

Distribution of anomaly Loss

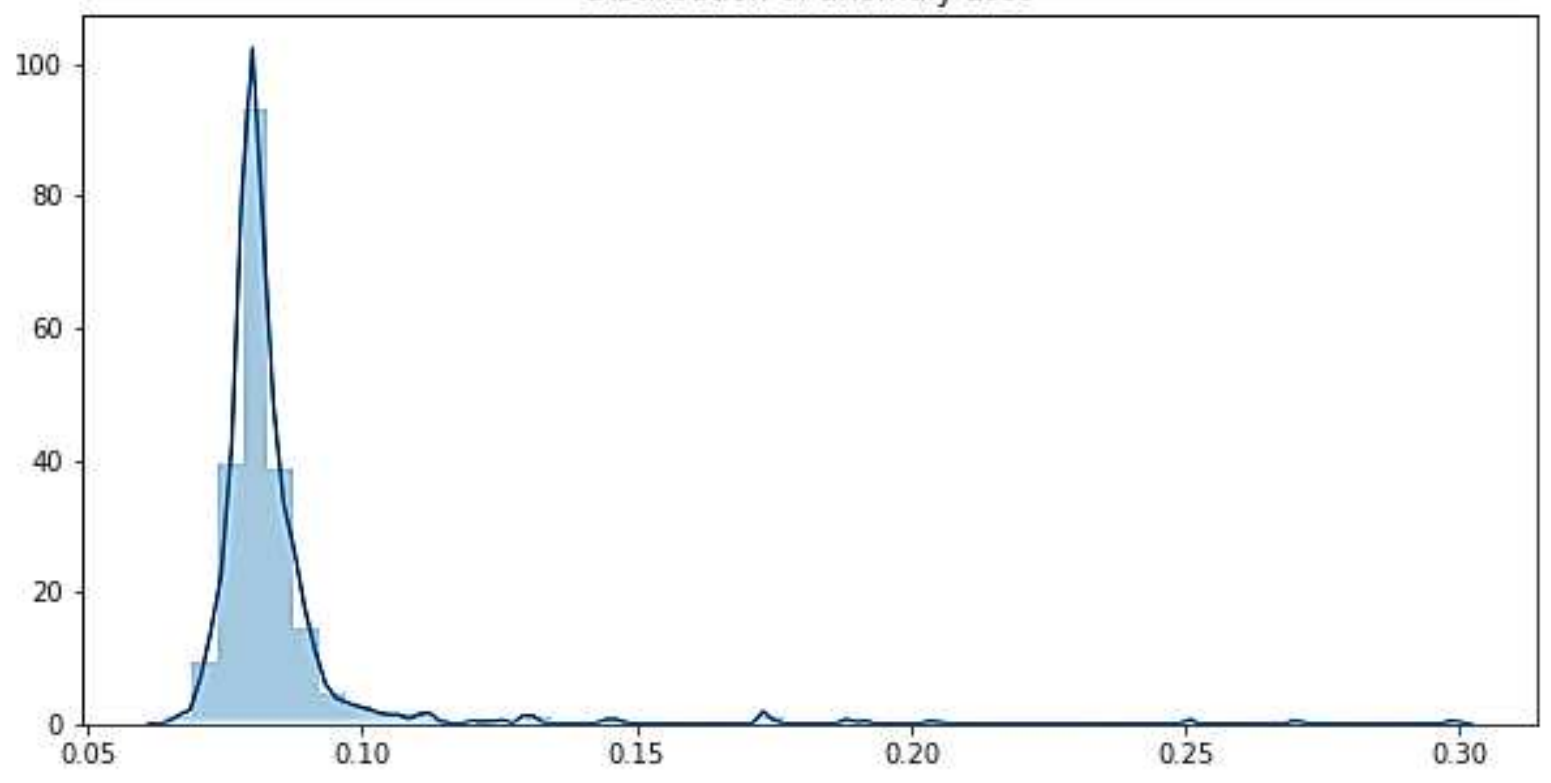

Figure 24

Distribution of Anomaly signals Loss 

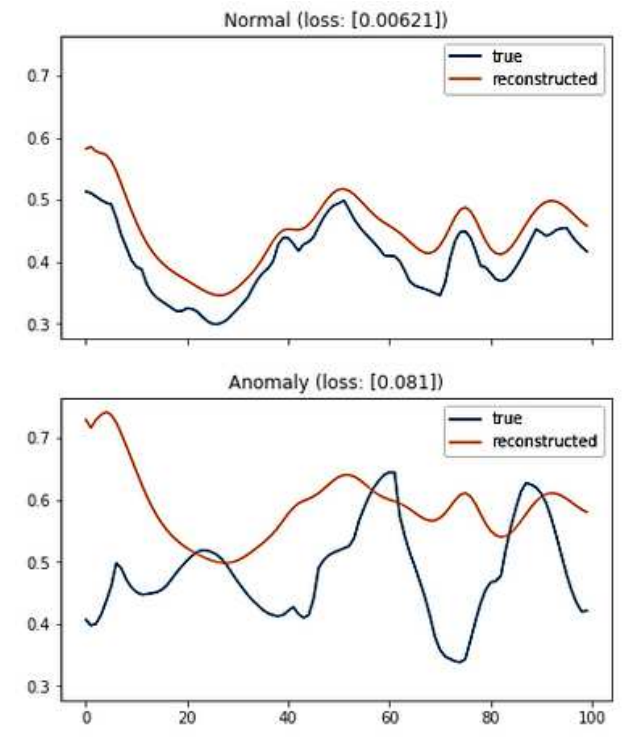

Normal (loss: [0.00692])

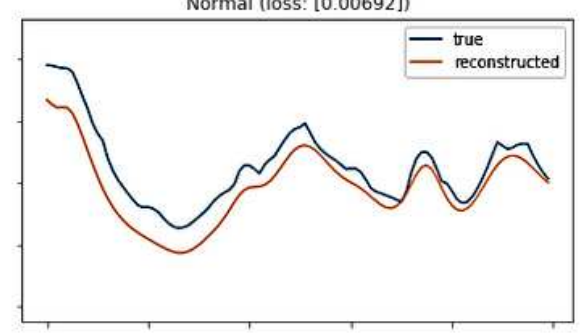

Anomaly (loss: [0.079])

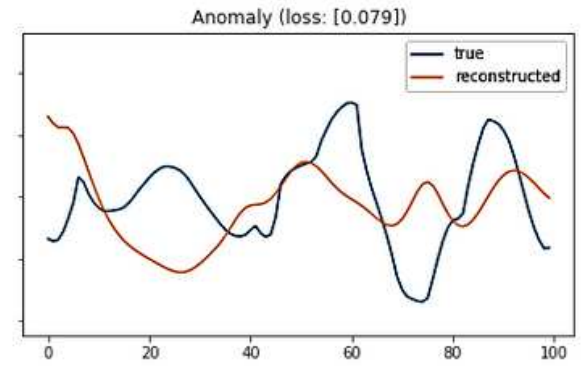

Normal (loss: [0.00839])

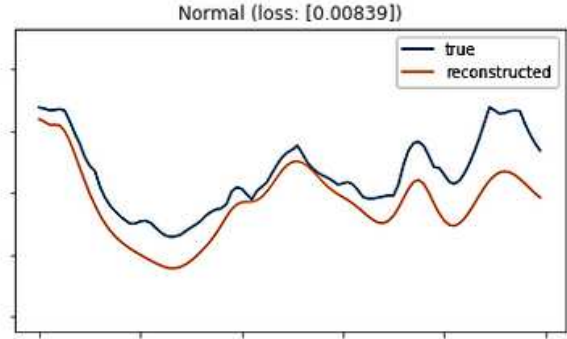

Anomaly (loss: [0.081])

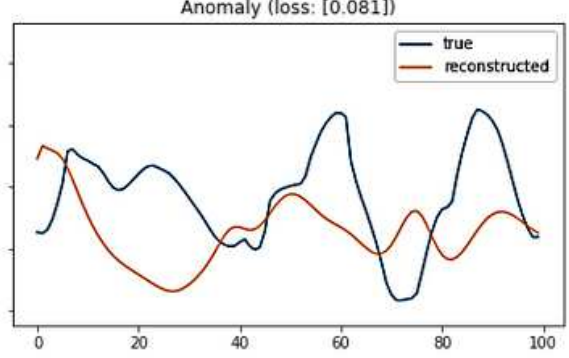

Figure 25

Reconstruction error of Normal \& Anomaly Signals 